\title{
Self-Dual Morphological Operators and Filters
}

\author{
HENK J.A.M. HEIJMANS \\ CWI, P.O. Box 94079, 1090 GB Amsterdam, The Netherlands
}

\begin{abstract}
The median operator is a nonlinear image transformation celebrated for its noise cleaning capacities. It treats the foreground and background of an image identically, i.e., it is self-dual. Unfortunately, the median operator has one major drawback: it is not idempotent. Even worse, subsequent iterations of a given image may lead to oscillations. This paper describes a general method for the construction of morphological operators which are self-dual. This construction is based upon the concept of a switch operator. Subsequently, the paper treats a class of operators, the so-called activity-extensive operators, which have the intriguing property that every sequence of iterates of a given image is pointwise monotone and therefore convergent. The underlying concept is that of the activity ordering. Every increasing, self-dual operator can be modified in such a way that it becomes activity-extensive. The sequence of iterates of this modification converges to a self-dual morphological filter.
\end{abstract}

Keywords: mathematical morphology, median operator, self-dual operator, idempotent operator, morphological filter, centre operator, activity ordering, activity-extensive operator, switch operator, persistent structure, iteration, cellular automata

\section{Introduction}

The median operator is likely to be one of the most frequently used transformations in image processing which is nonlinear. In fact, this operator (see Section 3 for a formal definition) fits quite well in the framework of mathematical morphology $[10,13,14,3]$; it is increasing, translation invariant, and can be decomposed as a finite union of erosions. The median operator is eminently suited for the elimination of noise [7]. Furthermore, it affects foreground and background noise in an identical manner. This is usually expressed by saying that the median operator is self-dual; see Section 2 for a formal definition. The major disadvantage of this operator is its non-idempotence: repeated application of the operator may change the outcome further or make previous changes undone. Even worse, iterating this operator may lead to oscillations. With regard to noise cleaning purposes, this is an undesirable property $[14,12]$.

Most of the operators discussed in this paper will be illustrated through their action on one particular noisy binary image $X$ with a resolution of $128 \times 128$ pixels; see the left-most image in Fig. 1. The second image in this figure shows $\mu(X)$, where $\mu$ is the median operator. The sequence of images obtained by iteration converges pixel-wise to a limit image $\mu^{\infty}(X)$; see the third image in Fig. 1. The right-most image shows all pixels which have been flipped at at least one iteration step; note that this set may be substantially larger than the symmetric difference of $X$ and $\mu^{\infty}(X)$.

Two other important operators known from mathematical morphology, are the opening and the closing. These operators have interesting noise cleaning capabilities, too, and they are idempotent. Unfortunately, however, they lack self-duality. As a matter of fact, opening and closing are complementary in the sense that the opening affects noise particles with high intensities, whereas the closing affects noise particles with low intensities.

Idempotence of an operator is a useful property as it guarantees that a second pass of this operator has no further effect on the image; for operators which are not idempotent, such as the median operator, it is not 

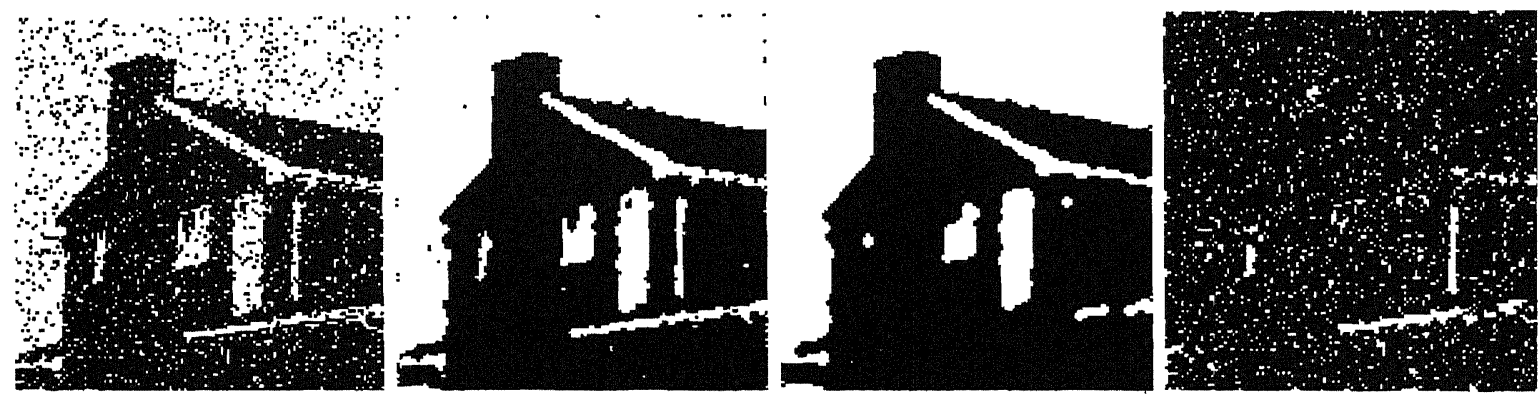

Figure 1. From left to right: the original image $X$, the transform $\mu(X)$, where $\mu$ is the median operator, the limit image $\mu^{\infty}(X)$, and the set of pixels which have been flipped at at least one iteration step.

known in advance how often it must be applied before the transformed image reaches a state which remains fixed or is periodic. Even worse, it is unknown in general if such a state will be reached at all.

In mathematical morphology the so-called Duality Principle plays an important role [3]. It says that every "morphological concept" has a dual counterpart, obtained by reversing the underlying partial ordering (set inclusion in the binary case). According to this principle, erosion is dual to dilation, opening is dual to closing, etc. On the level of (binary) images, this duality refers to the foreground and the background of an image. Erosion of the background of an image has the same effect as applying the dual (or negative) dilation to its foreground. An operator which treats foreground and background identically is called selfdual. Self-duality of an operator is a desirable property since it does not require an a priori distinction between what is foreground (what are the "objects") and what is background.

The major question addressed in this paper is the following: "Can we find a general method to design morphological operators which are self-dual and idempotent?" A partial answer to this question was given in our previous work [3, Chapter 13], [4]. In this paper we will review this work and supplement it with a substantial number of new results and examples.

The basic idea underlying our approach is to construct self-dual operators $\psi$ which are not necessarily idempotent, but which do satisfy the (weaker) constraint that they are activity-extensive. The latter means that the sequence of iterates $\psi, \psi^{2}, \psi^{3}, \ldots$ is increasing with respect to the so-called activity ordering on the set of all operators; this ordering was introduced by Serra [14] and will be reviewed in Section 2. If $\psi$ is an activity-extensive operator, then repeated application of $\psi$ to an image yields a convergent sequence. This leaves us with the problem of finding self-dual operators which are activity-extensive. This part of the problem will constitute the main body of the present paper.

In Section 2 we recall the basic concepts which we need in this paper. As much as possible we adopt the terminology and notation of [3]. We point out that we use the terminology "operator" for an arbitrary transformation; the word "filter" is preserved for operators which are increasing and idempotent. In Section 3 we will make some general statements about self-dual operators; in particular, we will recall the notions of a (weighted) rank and median operator. We also establish a lattice isomorphism between the self-dual operators provided with the activity ordering at the one hand, and the anti-extensive operators with the pointwise ordering at the other. In Section 4 we define switch operators and indicate their importance in relation to our problem of finding increasing, self-dual operators. We study an important instance of a switch operator corresponding with the situation that we want to eliminate isolated noise points. Here the word "isolated" has to be interpreted in terms of some prescribed adjacency relation on the underlying point set. From Section 5 onwards, we will restrict attention to operators which are translation invariant. Under this restriction we can give a complete characterization of switch operators, and hence, of increasing, self-dual operators. Subsequently, in Section 6 we show how an arbitrary increasing, self-dual operator can be modified such that it becomes activity-extensive; then this modification can be used to construct a self-dual filter by iteration. A large number of examples will be presented in Section 7 . We end with some conclusions in Section 8; there we will also point out some open problems. 


\section{Terminology and Basic Facts}

We presume that the reader is familiar with the basic notions of mathematical morphology. Throughout this paper we are mainly concerned with the space of discrete binary images $\mathcal{P}\left(\mathbb{Z}^{2}\right)$. If $\phi, \psi$ are two operators on this space, then $\phi \leq \psi$ means $\phi(X) \subseteq \psi(X)$, for every $X \subseteq \mathbb{Z}^{2}$. Furthermore, $\psi \phi$ denotes the composed operator given by $(\psi \phi)(X)=\psi(\phi(X))$. If $\psi_{i}$, $i \in I$, is a collection of operators then $\bigwedge_{i \in I} \psi_{i}$ and $V_{i \in I} \psi_{i}$ denote the infimum and supremum of this collection, respectively; see e.g., [3].

If $X \subseteq \mathbb{Z}^{2}$ and $h \in \mathbb{Z}^{2}$ then $X_{h}$ denotes the translation of the set $X$ along the vector $h$. Sometimes we interpret $X$ as an indicator function. Then $X(h)=1$ if $h \in X$ and 0 otherwise. Given a translation invariant operator $\psi$ on $\mathcal{P}\left(\mathbb{Z}^{2}\right)$, we say that $\psi$ is a finite-window operator if there exists a finite set $M \subseteq \mathbb{Z}^{2}$ such that $h \in \psi(X) \Longleftrightarrow h \in \psi\left(X \cap A_{h}\right)$ for every $h \in \mathbb{Z}^{2}$, $X \subseteq \mathbb{Z}^{2}$ and $A \supseteq M$. If $\psi$ is increasing then it suffices to consider $A=M$.

In [14] Serra introduces a partial ordering on the complete lattice of all operators on $\mathcal{L}$, where $\mathcal{L}$ is a complete Boolean lattice. Here we specialize to the case $\mathcal{L}=\mathcal{P}\left(\mathbb{Z}^{2}\right)$. Given two operators $\phi, \psi$ on $\mathcal{P}\left(\mathbb{Z}^{2}\right)$, we say that $\psi$ is more active than $\phi$, written $\phi \preccurlyeq \psi$, if

$$
\text { id } \wedge \psi \leq \mathrm{id} \wedge \phi \quad \text { and } \quad \text { id } \vee \psi \geq \mathrm{id} \vee \phi
$$

Here id is the identity operator which maps every set onto itself. The infimum of a collection of operators $\psi_{i}, i \in I$, with respect to the activity ordering is denoted by $\curlywedge_{i \in I} \psi_{i}$. It is given by the expression

$$
\hat{\curlywedge}_{i \in I} \psi_{i}=\left(\mathrm{id} \wedge \bigvee_{i \in I} \psi_{i}\right) \vee \bigwedge_{i \in I} \psi_{i}
$$

This operator is sometimes called the centre of the operators $\psi_{i}$. Dually, their supremum, denoted by $\gamma_{i \in I} \psi_{i}$, is given by

$$
\underset{i \in I}{\curlyvee} \psi_{i}=\left(\nu \wedge \bigvee_{i \in I} \psi_{i}\right) \vee \bigwedge_{i \in I} \psi_{i}
$$

and is called the anti-centre. Here $\nu$ is the complement operator given by

$$
\nu(X)=X^{c}
$$

It is obvious that id and $\nu$ are the least and greatest operator, respectively, with respect to the activity ordering. The negative $\psi^{*}$ of an operator $\psi$ is given by $\psi^{*}=\nu \psi \nu$, i.e., $\psi^{*}(X)=\left(\psi\left(X^{c}\right)\right)^{c}$.

The operator $\psi$ is called activity-extensive if

$$
\psi^{n} \preccurlyeq \psi^{n+1}, \quad n=0,1,2, \ldots
$$

This means that, for a fixed set $X \subseteq \mathbb{Z}^{2}$, the sequence $\psi^{n}(X)$ is pointwise monotone: the sequence $\psi^{n}(X)(h)$, where $h \in \mathbb{Z}^{2}$ is given, is monotone, either increasing or decreasing. As such sequences consist exclusively of 0 's and 1's, they must be of the form $0,0, \ldots, 0,1,1, \ldots$ or $1,1, \ldots, 1,0,0, \ldots$ We call $\left\{\psi^{n}(X) \mid n=0,1,2, \ldots\right\}$ the orbit starting at $\psi^{0}(X)=X$.

\section{Self-Dual Operators}

Before entering a general discussion on the construction of self-dual operators we present some examples based on Boolean functions; see also [3, Section 4.5]. Let $A=\left\{a_{1}, a_{2}, \ldots, a_{n}\right\}$ be a finite structuring element, and let $b$ be the threshold function with realization vector $\left(w_{1}, \ldots, w_{n} \mid s\right)$, i.e.,

$$
b\left(u_{1}, \ldots, u_{n}\right)=\left[\sum_{i=1}^{n} w_{i} u_{i} \geq s\right]
$$

here $w_{1}, \ldots, w_{n}, s \in \mathbb{Z}$. Here we use the following convention. The expression $[P]$ equals 1 if $P$ is true and 0 if $P$ is false. Now

$$
\psi_{b}(X)=\left\{x \in \mathbb{Z}^{2} \mid \sum_{i=1}^{n} w_{i} X\left(x+a_{i}\right) \geq s\right\}
$$

is an increasing, translation invariant, morphological operator. We call this operator weighted rank operator. If the weights $w_{i}$ as well as the threshold $s$ are positive integers, then this operator has the following interpretation: the point $x$ lies in $\psi_{b}(X)$ if the $s$ th value of the sequence which is obtained by putting the values $X\left(x+a_{i}\right)$ (counted $w_{i}$ times) in decreasing order equals one. If $w_{i} \geq 0$ for every $i$, then $\psi_{b}$ is an increasing operator. Its negative $\psi_{b}^{*}$ is given by

$$
\psi_{b}^{*}(X)=\left\{x \in \mathbb{Z}^{2} \mid \sum_{i=1}^{n} w_{i} X\left(x+a_{i}\right) \geq \sum_{i=1}^{n} w_{i}-s+1\right\}
$$


It follows immediately that $\psi_{b}$ is self-dual if and only if $2 s=\sum_{i=1}^{n} w_{i}+1$. In this case $\psi_{b}$ is called a weighted median operator. A weighted rank operator can be represented by a matrix containing the weights $w_{i}$ in which the position of the origin is marked; see Example 3.1 below for an illustration.

If all weights $w_{i}$ have the value 1 , and if $s \in$ $\{1,2, \ldots, n\}$, then $\psi_{b}$ is called rank operator; this operator is denoted by $\rho_{A, s}$. It is easy to show that $h \in \rho_{A, s}(X)$ if and only if $X \cap A_{h}$ has at least cardinality $s$. In particular,

$$
\rho_{A, 1}(X)=X \oplus \check{A} \quad \text { and } \quad \rho_{A, n}(X)=X \ominus A .
$$

Furthermore,

$$
\rho_{A, n} \leq \rho_{A, n-1} \leq \cdots \leq \rho_{A, 1}
$$

If $n$ is odd and $s=(n+1) / 2$ then $\rho_{A, s}$ is self-dual; we use the notation

$$
\mu_{A}=\rho_{A,(n+1) / 2}
$$

if $n$ is odd, and we call $\mu_{A}$ the median operator.

Let $\psi$ be an operator on $\mathcal{P}\left(\mathbb{Z}^{2}\right)$ with negative $\psi^{*}$.
The centre of $\psi$ and $\psi^{*}$ is given by

$$
\gamma=\left(\mathrm{id} \wedge\left(\psi \vee \psi^{*}\right)\right) \vee\left(\psi \wedge \psi^{*}\right)
$$

The anti-centre is

$$
\kappa=\left(\nu \wedge\left(\psi \vee \psi^{*}\right)\right) \vee\left(\psi \wedge \psi^{*}\right)
$$

where $v$ is the complement operator. It is easy to see that both $\gamma$ and $\kappa$ are self-dual. Furthermore, $\gamma$ is increasing if $\psi$ is increasing.

Example 3.1. Let $A$ be the $3 \times 3$ square centred at the origin, and let $\rho_{A, s}, 1 \leq s \leq 9$, be the associated rank operators. The negative of $\rho_{A, s}$ is $\rho_{A, 10-s}$, and $\rho_{A, s} \leq \rho_{A, 10-s}$ if $s \geq 5$. Define $\theta_{s}$ as the centre of $\rho_{A, s}$ and $\rho_{A, 10-s}$; hence

$$
\theta_{s}=\left(\mathrm{id} \wedge \rho_{A, 10-s}\right) \vee \rho_{A, s}, \quad s=5,6,7,8,9 .
$$

Note that $\theta_{5}=\mu_{A}$, the median operator. Obviously, every $\theta_{s}$ is self-dual. Furthermore, it is easy to show that

$$
\mathrm{id}=\theta_{9} \preccurlyeq \theta_{8} \preccurlyeq \theta_{7} \preccurlyeq \theta_{6} \preccurlyeq \theta_{5} .
$$

In Fig. 2 the action of these operators is illustrated by means of an example. It is easy to show that $\theta_{k}$

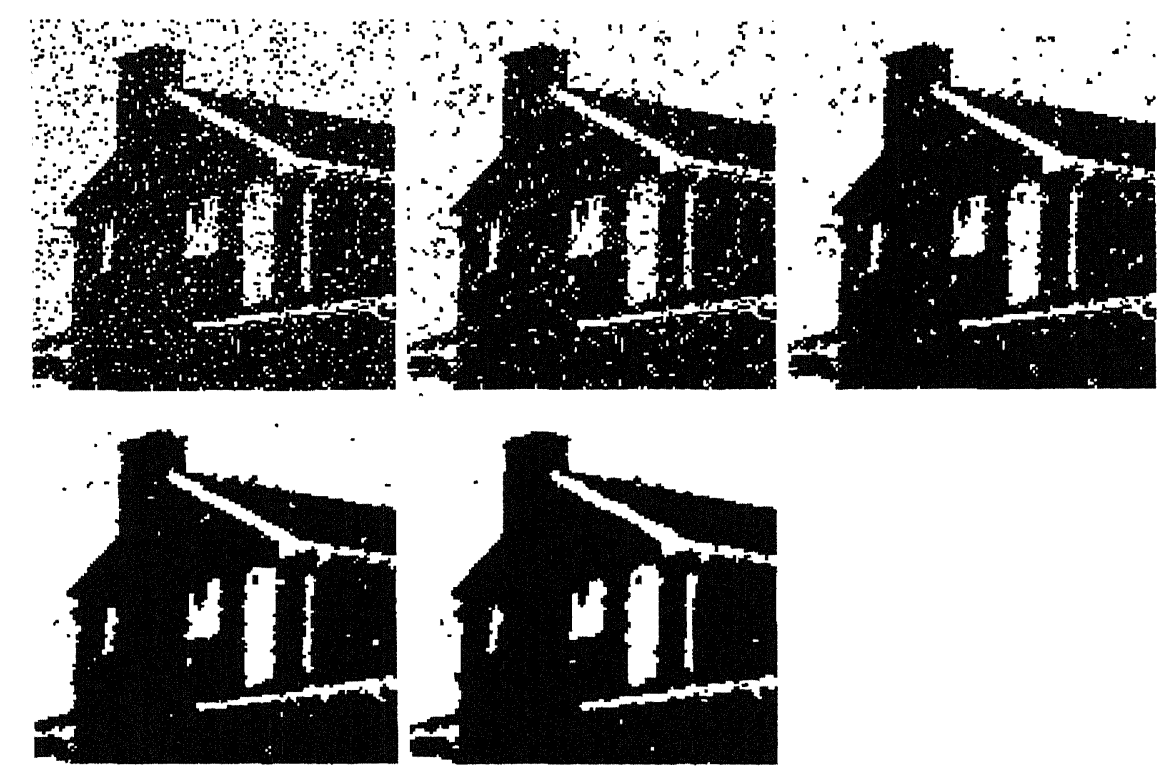

Figure 2. From left to right and top to bottom: original image $X$ and its transforms $\theta_{8}(X), \theta_{7}(X), \theta_{6}(X), \theta_{5}(X)$. This picture illustrates nicely that $\theta_{8} \preccurlyeq \theta_{7} \preccurlyeq \theta_{6} \preccurlyeq \theta_{5}$. 
corresponds with the weighted median operator represented by the matrix

$$
w=\left(\begin{array}{ccc}
1 & 1 & 1 \\
1 & 2 k-9 & 1 \\
1 & 1 & 1
\end{array}\right)
$$

the centre of the matrix corresponds with the origin, and the threshold $s$ equals $k$.

Let us have a closer look at the median operator $\mu_{A}$ defined in the previous example. When a point $h$ lies in the complement of $X$, then $h \in \mu_{A}(X)$ if it is surrounded by at least five neighbours which lie in $X$ like in one of the following 56 configurations:

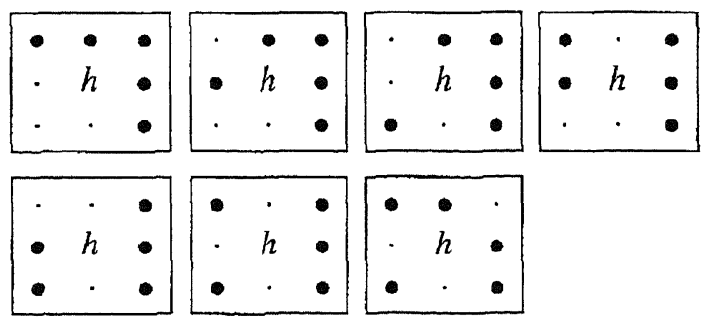

Note that there correspond eight rotations with every structuring element depicted. Dually, when $h$ lies in $X$, then $h$ lies outside $\mu_{A}(X)$ if it has at least five neighbours in the complement of $X$.

Consider an arbitrary self-dual operator $\psi$. We denote the points which are contained in $X$ but not in $\psi(X)$ by $\sigma(X)$. Hence

$$
\begin{aligned}
\sigma(X) & =X \backslash \psi(X)=X \cap(\psi(X))^{c} \\
& =X \cap \psi\left(X^{c}\right) .
\end{aligned}
$$

Here we have used that $\psi$ is self-dual, hence

$$
\psi\left(X^{c}\right)=(\psi(X))^{c}
$$

It is easy to check that $\psi$ can be recovered from $\sigma$ by means of the formula:

$$
\psi(X)=(X \backslash \sigma(X)) \cup \sigma\left(X^{c}\right)
$$

Motivated by the above observations we define two mappings on $\mathcal{O}$, the lattice of operators on $\mathcal{P}\left(\mathbb{Z}^{2}\right)$.

Definition 3.2. Let the mappings $\Sigma, \Psi: \mathcal{O} \rightarrow \mathcal{O}$ be defined by

$$
\Sigma(\psi)=\mathrm{id} \wedge \psi \nu, \quad \psi \in \mathcal{O}
$$

$$
\Psi(\sigma)=(\mathrm{id} \wedge \nu \sigma) \vee \sigma \nu, \quad \sigma \in \mathcal{O}
$$

Observe that (3.1) and (3.2) can be reformulated as $\sigma=\Sigma(\psi)$ and $\psi=\Psi(\sigma)$, respectively. Furthermore

$$
\sigma \leq \mathrm{id} \Longleftrightarrow \sigma \nu \leq \nu \Longleftrightarrow v \leq \nu \sigma,
$$

for every operator $\sigma$. This means in particular that $\Psi(\sigma)$ is the centre of $\nu \sigma$ and $\sigma \nu$ if $\sigma$ is anti-extensive.

We denote by $\mathcal{O}_{\text {sd }}$ and $\mathcal{O}_{\text {ae }}$ the self-dual and antiextensive operators on $\mathcal{P}\left(\mathbb{Z}^{2}\right)$, respectively. It is obvious that $\left(\mathcal{O}_{\mathrm{ae}}, \leq\right)$ is a complete sublattice of $(\mathcal{O}, \leq)$. From the fact that both the centre and the anti-centre of a family of self-dual operators is self-dual (cf. [3, Prop. 3.42]), we conclude that $\left(\mathcal{O}_{\text {sd }}, \preccurlyeq\right)$ is a complete sublattice of $(\mathcal{O}, \preccurlyeq)$.

\section{Proposition 3.3.}

(a) $\Sigma:\left(\mathcal{O}_{s d}, \preccurlyeq\right) \rightarrow\left(\mathcal{O}_{a e}, \leq\right)$ is increasing.

(b) $\Psi:\left(\mathcal{O}_{a e}, \leq\right) \rightarrow\left(\mathcal{O}_{s d}, \preccurlyeq\right)$ is increasing.

\section{Proof:}

(a) It is evident that $\Sigma(\psi)$ is anti-extensive. Let $\psi_{1}, \psi_{2}$ be self-dual operators and $\psi_{1} \preccurlyeq \psi_{2}$. Then id $\vee$ $\psi_{1} \leq$ id $\vee \psi_{2}$, hence $v \vee \psi_{1} v=$ (id $\left.\vee \psi_{1}\right) v \leq$ (id $\left.\vee \psi_{2}\right) v=v \vee \psi_{2} v$. Taking the infimum with id we get id $\wedge\left(\nu \vee \psi_{1} v\right) \leq$ id $\wedge\left(\nu \vee \psi_{2} \nu\right)$, that is, id $\wedge \psi_{1} \nu \leq$ id $\wedge \psi_{2} \nu$.

(b) First we must show that $\Psi(\sigma)$ is self-dual if $\sigma \leq$ id. This is done as follows:

$$
\begin{aligned}
\Psi(\sigma)^{*} & =\nu \Psi(\sigma) \nu \\
& =\nu((i d \wedge \nu \sigma) \vee \sigma \nu) \nu \\
& =\nu((\nu \wedge \nu \sigma \nu) \vee \sigma) \\
& =\nu(\nu \wedge \nu \sigma \nu) \wedge \nu \sigma \\
& =(i d \vee \sigma \nu) \wedge \nu \sigma \\
& =(\text { id } \wedge \nu \sigma) \vee \sigma \nu \\
& =\Psi(\sigma) ;
\end{aligned}
$$

here we have used that $\sigma \nu \leq \nu \sigma$ by the antiextensivity of $\sigma$. To show that $\Psi$ is increasing, let $\sigma_{1} \leq \sigma_{2} \leq \mathrm{id}$, and let $\Psi\left(\sigma_{1}\right)=\psi_{1}, \Psi\left(\sigma_{2}\right)=\psi_{2}$. We show that $\psi_{1} \preccurlyeq \psi_{2}$. First,

$$
\text { id } \begin{aligned}
\wedge \psi_{1} & =\text { id } \wedge\left(\left(\mathrm{id} \wedge \nu \sigma_{1}\right) \vee \sigma_{1} \nu\right) \\
& =\left(\mathrm{id} \wedge\left(\mathrm{id} \wedge \nu \sigma_{1}\right)\right) \vee\left(\mathrm{id} \wedge \sigma_{1} \nu\right)
\end{aligned}
$$




$$
\begin{aligned}
& =\left(\mathrm{id} \wedge \nu \sigma_{1}\right) \vee\left(\mathrm{id} \wedge \sigma_{1} \nu\right) \\
& =\mathrm{id} \wedge \nu \sigma_{1},
\end{aligned}
$$

since $\nu \sigma_{1} \geq \sigma_{1} \nu$ by (3.5). Now $\sigma_{1} \leq \sigma_{2}$ implies that id $\wedge \psi_{1} \geq$ id $\wedge \psi_{2}$. We also find

$$
\mathrm{id} \vee \psi_{1}=\mathrm{id} \vee \sigma_{1} \nu
$$

Clearly, $\sigma_{1} \leq \sigma_{2}$ implies that id $\vee \psi_{1} \leq \mathrm{id} \vee \psi_{2}$. Thus we find that $\psi_{1} \preccurlyeq \psi_{2}$.

Proposition 3.4. The mappings $\Sigma:\left(\mathcal{O}_{s d}, \preccurlyeq\right) \rightarrow$ $\left(\mathcal{O}_{a e}, \leq\right)$ and $\Psi:\left(\mathcal{O}_{a e}, \leq\right) \rightarrow\left(\mathcal{O}_{s d}, \preccurlyeq\right)$ are bijective (onto and one-one). Furthermore, these mappings are each other's inverses, i.e.,

$$
\begin{aligned}
& \Sigma(\Psi(\sigma))=\sigma, \text { for } \quad \sigma \in \mathcal{O}_{a e} \\
& \Psi(\Sigma(\psi))=\psi, \text { for } \quad \psi \in \mathcal{O}_{s d} .
\end{aligned}
$$

Proof: We prove (3.6) and leave (3.7) as an exercise for the reader. Note that the validity of these two relations implies the bijectivity of the mappings $\Sigma$ and $\Psi$.

Let $\sigma$ be anti-extensive; then

$$
\begin{aligned}
\Sigma(\Psi(\sigma)) & =\mathrm{id} \wedge \Psi(\sigma) \nu=\mathrm{id} \wedge((\mathrm{id} \wedge \nu \sigma) \vee \sigma \nu) \nu \\
& =\mathrm{id} \wedge((\nu \wedge \nu \sigma \nu) \vee \sigma) \\
& =(\mathrm{id} \wedge \nu \wedge \nu \sigma \nu) \vee(\mathrm{id} \wedge \sigma) \\
& =\mathrm{id} \wedge \sigma=\sigma .
\end{aligned}
$$

Here we have used that $\mathrm{id} \wedge \nu=0$, the operator which maps every set onto the empty set.

The following relations are easily derived from the previous result.

$$
\begin{aligned}
& \Sigma\left(\gamma_{i \in I} \psi_{i}\right)=\bigvee_{i \in I} \Sigma\left(\psi_{i}\right) \\
& \Sigma\left(\curlywedge_{i \in I} \psi_{i}\right)=\bigwedge_{i \in I} \Sigma\left(\psi_{i}\right) \\
& \Psi\left(\bigvee_{i \in I} \sigma_{i}\right)=\underset{i \in I}{\curlyvee} \Psi\left(\sigma_{i}\right) ; \\
& \Psi\left(\bigwedge_{i \in I} \sigma_{i}\right)=\curlywedge_{i \in I} \Psi\left(\sigma_{i}\right) .
\end{aligned}
$$

Here we prove (3.8). Let $\psi_{i}$ be self-dual operators for $i \in I$, and let $\sigma_{i}=\Sigma\left(\psi_{i}\right)$. Since $\psi_{j} \preccurlyeq Y_{i \in I} \psi_{i}$ for $j \in I$, we obtain $\sigma_{j} \leq \Sigma\left(\Upsilon_{i \in I} \psi_{i}\right)$. This implies that $\bigvee_{i \in I} \sigma_{i} \leq \Sigma\left(\Upsilon_{i \in I} \psi_{i}\right)$, hence that $\sigma_{j} \leq \bigvee_{i \in I} \sigma_{i} \leq$ $\Sigma\left(\Upsilon_{i \in I} \psi_{i}\right)$. Applying $\Psi$ we arrive at

$$
\psi_{j} \preccurlyeq \Psi\left(\bigvee_{i \in I} \sigma_{i}\right) \preccurlyeq \underset{i \in I}{\curlyvee} \psi_{i} .
$$

Taking the activity supremum over all $j$ at the left-hand side, we get

$$
\underset{i \in I}{\curlyvee} \psi_{i} \preccurlyeq \Psi\left(\bigvee_{i \in I} \sigma_{i}\right) \preccurlyeq \underset{i \in I}{\curlyvee} \psi_{i} .
$$

But then all three terms must be equal, meaning that

$$
\underset{i \in I}{\curlyvee} \psi_{i}=\Psi\left(\bigvee_{i \in I} \sigma_{i}\right)
$$

Applying $\Sigma$ on both sides and using that $\Sigma \Psi=\mathrm{id}$, gives

$$
\Sigma\left(\underset{i \in I}{\gamma} \psi_{i}\right)=\bigvee_{i \in I} \Sigma\left(\psi_{i}\right)
$$

The least and greatest operator with respect to the activity ordering are id and $\nu$, respectively. It is easy to check that $\Sigma$ (id) $=o$ and $\Sigma(\nu)=$ id, the least resp. greatest anti-extensive operator.

Remark 3.5. Let $\psi$ be an operator, not necessarily self-dual. The centre $\psi \curlywedge \psi^{*}$ and the anti-centre $\psi \curlyvee \psi^{*}$ are self-dual. A straightforward calculation shows that

$$
\begin{aligned}
& \Sigma\left(\psi \curlywedge \psi^{*}\right)=\Sigma\left(\psi \wedge \psi^{*}\right)=\mathrm{id} \wedge(\psi \nu \wedge \nu \psi) \\
& \Sigma\left(\psi \curlyvee \psi^{*}\right)=\Sigma\left(\psi \vee \psi^{*}\right)=\operatorname{id} \wedge(\psi \nu \vee \nu \psi)
\end{aligned}
$$

The results given so far provide a general method to construct self-dual operators. We give some special attention to the class of translation invariant operators. It is a well-known fact [1], [3, Section 4.2] that every translation invariant operator $\psi$ can be decomposed as a union of hit-or-miss operators. Recall that the hitor-miss operator with structuring elements $A, B$ is the $\operatorname{map} X \mapsto X \circledast(A, B)$, where

$$
X \otimes(A, B)=\left\{h \in \mathbb{Z}^{2} \mid A_{h} \subseteq X, B_{h} \subseteq X^{c}\right\} .
$$

In order that $X \otimes(A, B) \subseteq X$ for every $X$ we must demand that $0 \in A$. Thus we may conclude that every 

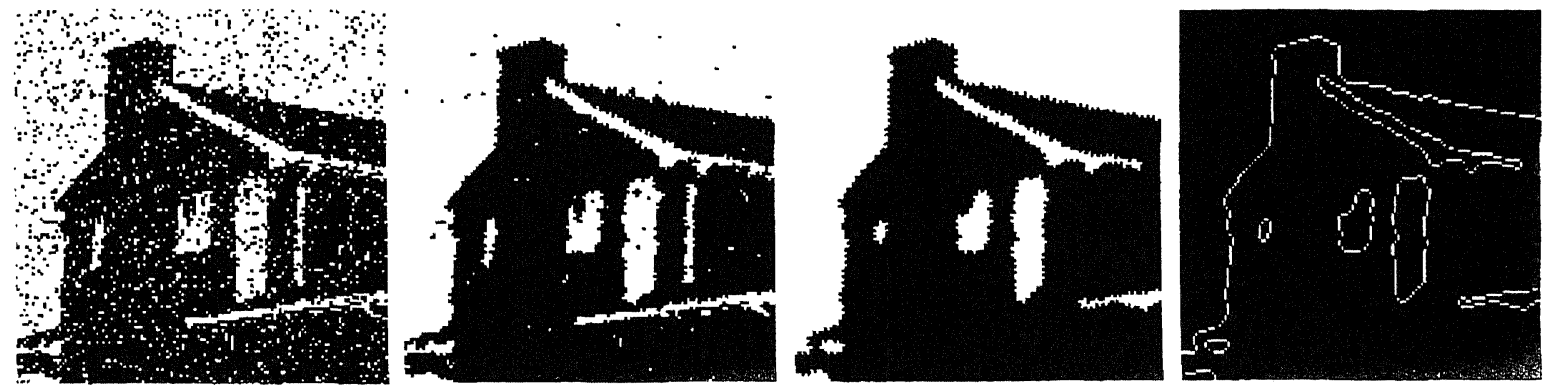

Figure 3. The first image is $X$, the second $\psi(X)$, with $\psi$ as defined in Example 3.6. The sequence $\psi^{n}(X)$ converges to a period-2 orbit containing two images. The third image represents one of these two images $Y$. The right-most image shows the symmetric difference $Y \Delta \psi(Y)$.

anti-extensive operator $\sigma$ can be written as a union of hit-or-miss operators $X \mapsto X \circledast(A, B)$ with $0 \in A$.

Example 3.6. Consider the following eight structuring elements ( $A$ contains the central black pixel, $B$ contains the white pixels) respectively called $\left(A_{1}, B_{1}\right), \ldots,\left(A_{8}, B_{8}\right)$. The underlined (central) pixel is located at the origin.

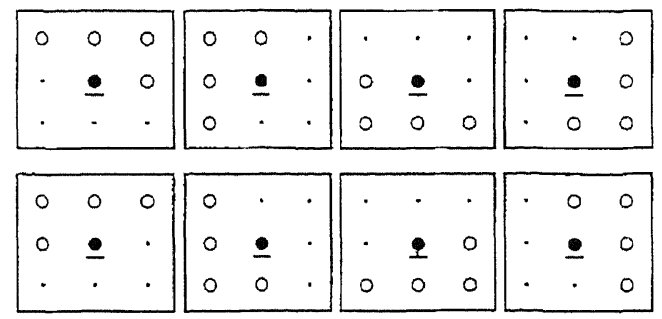

In [3, Example 4.6] these same patterns are used to obtain the pseudo-convex hull of a discrete set by iterations of the corresponding thickenings $X \odot(A, B)=$ $X \cup X \otimes(A, B)$. Here we define

$$
\sigma(X)=\bigcup_{k=1}^{8} X \otimes\left(A_{k}, B_{k}\right)
$$
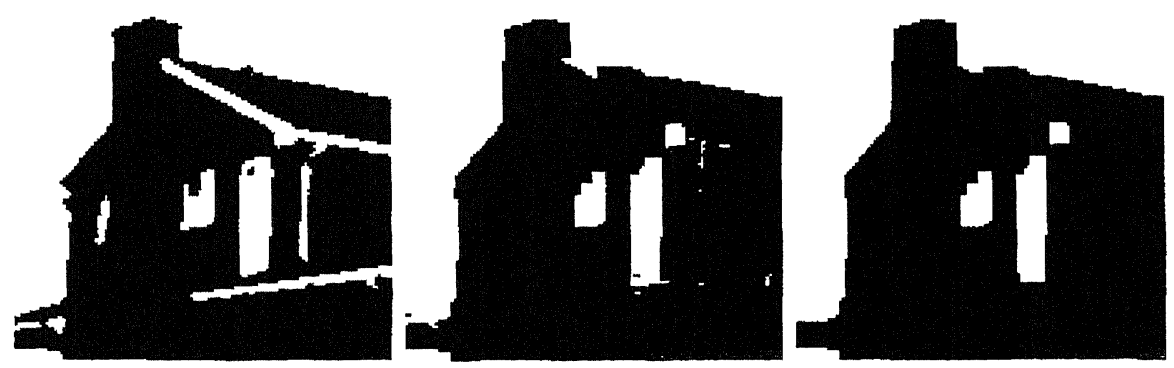

Figure 4. From left to right: the original image $X$, the transformed image $\psi(X)$, and the limit $\psi^{\infty}(X)$. Here $\psi=\alpha \curlyvee \beta$, where $\alpha$ is the structural opening by the $3 \times 3$ square and $\beta$ is the negative closing.
Note that $\sigma \leq$ id. Let $\psi=\Psi(\sigma)$ be the corresponding self-dual operator. In Fig. 3 we depict an image $X$ and its transform $\psi(X)$. If we compute the sequence of iterates $\psi^{n}(X)$ we find that this sequence converges to a period-2 orbit consisting of two images $Y$ and $\psi(Y)$. In Fig. 3 we depict $Y$ and the symmetric difference $Y \triangle \psi(Y)$.

Example 3.7. Let $\alpha$ be an opening and $\sigma(X)=$ $X \backslash \alpha(X)$; in other words, $\sigma=\mathrm{id} \wedge \nu \alpha$. The corresponding self-dual operator $\psi=\Psi(\sigma)$ equals $\psi=$ $(\nu \wedge \beta) \vee \alpha=(v \vee \alpha) \wedge \beta$, where $\beta$ is the negative of $\alpha$. Note that $\psi$ is the anti-centre of $\alpha$ and $\beta$, i.e., $\psi=\alpha \curlyvee \beta$. In Fig. 4 we have illustrated $\alpha \curlyvee \beta$, where $\alpha$ is the translation invariant, structural opening by the $3 \times 3$ square. The first image is the original image $X$, the second image is $\psi(X)$. In this particular case, the sequence $\psi^{n}(X)$ tends to a limit $\psi^{\infty}(X)$ as $n \rightarrow \infty$; this is the right-most image in Fig. 4.

There do also exist images $Y$ such that $\psi^{n}(Y)$ converges to a period-2 orbit. For example, if $Y$ is such that $\alpha(Y)=\alpha\left(Y^{c}\right)=\emptyset$, then $\sigma(Y)=Y$ and $\sigma\left(Y^{c}\right)=Y^{c}$. Thus $\psi(Y)=Y^{c}$ and $\psi\left(Y^{c}\right)=Y$. This means that the pair $\left\{Y, Y^{c}\right\}$ forms a period- 2 orbit. 


\section{Switch Operators}

The self-dual operators $\psi$ in Examples 3.6-3.7 are not increasing. In this section we will answer the following question: which conditions must $\sigma$ satisfy in order that the corresponding self-dual operator $\psi=\Psi(\sigma)$ is increasing. Assume that $X \subseteq Y$. From the interpretation of $\sigma(X)$ as the points in $X$ which are deleted by $\psi$ we make the following observations. If a point $h \in X$ is not contained in $\sigma(X)$ then it must not be contained in $\sigma(Y)$, for otherwise $h \in \psi(X)$ but $h \notin \psi(Y)$. This leads us to the following condition:

(i) $h \in X, h \notin \sigma(X) \Rightarrow h \notin \sigma(Y)$.

A similar condition must hold for background points:

(ii) $h \in Y^{c}, h \notin \sigma\left(Y^{c}\right) \Rightarrow h \notin \sigma\left(X^{c}\right)$.

Suppose, finally, that a point $h$ lies in $Y$ but not in $X$. To avoid that $h$ is mapped out of $Y$ and at the same time into $X$ we must assume:

(iii) $h \in \sigma\left(X^{c}\right) \Rightarrow h \notin \sigma(Y)$.

It is easy to see that (i)-(iii) amount to the following conditions:

(S1) $X \subseteq Y$ implies that $X \cap \sigma(Y) \subseteq \sigma(X)$.

$(\mathrm{S} 2) \sigma(X \cup\{h\}) \cap \sigma\left(X^{c} \cup\{h\}\right)=\emptyset, \quad h \in \mathbb{Z}^{2}, X \subseteq \mathbb{Z}^{2}$.

We show that (iii) follows from (S1)-(S2); note, however, that (iii) is not equivalent to (S2). Assume that $h \in \sigma\left(X^{c}\right)$ and $h \in \sigma(Y)$; we must show that this leads to a contradiction. From (S2) we conclude that $h \notin \sigma(X \cup\{h\})$. As $X \cup\{h\} \subseteq Y$ we conclude from (S1) that

$$
(X \cup\{h\}) \cap \sigma(Y) \subseteq \sigma(X \cup\{h\}) .
$$

But then $h \in \sigma(X \cup\{h\})$, a contradiction.

Definition 4.1. An anti-extensive operator $\sigma$ is called a switch operator if (S1) and (S2) hold.

The adjective "switch" indicates that the operator $\sigma$ yields all points which switch value from 1 to 0 (points in $\sigma(X)$ ) or from 0 to 1 (points in $\sigma\left(X^{c}\right)$ ) by application of the self-dual operator $\psi=\Psi(\sigma)$.

In the previous paragraphs we have established the following result.
Theorem 4.2. Let $\sigma$ be anti-extensive and $\psi=$ $\Psi(\sigma)$; then $\psi$ is increasing if and only if $\sigma$ is a switch operator.

If $\psi$ is a self-dual increasing operator, and $\eta$ is increasing and extensive, then

$$
\psi^{\prime}=(\mathrm{id} \wedge \psi \eta) \vee \psi \eta^{*}
$$

is the centre of $\psi \eta$ and its negation $\psi \eta^{*}$; note that $\eta^{*} \leq \eta$. Therefore, $\psi^{\prime}$ is self-dual and increasing too. The following result expresses the corresponding switch operator $\Sigma\left(\psi^{\prime}\right)$ in terms of $\Sigma(\psi)$ and $\eta$.

Proposition 4.3. Let $\sigma$ be a switch operator, $\psi=$ $\Psi(\sigma)$, and let $\eta$ be increasing and extensive; then $\sigma^{\prime}=$ id $\wedge \sigma \eta$ is a switch operator too, and $\Psi\left(\sigma^{\prime}\right)=$ (id $\wedge$ $\psi \eta) \vee \psi \eta^{*}$. Furthermore, $\sigma^{\prime} \leq \sigma$ and $\psi^{\prime} \preccurlyeq \psi$.

Proof: We show that $\Psi\left(\sigma^{\prime}\right)=($ id $\wedge \psi \eta) \vee \psi \eta^{*}$, and therefore increasing. This yields automatically that $\sigma^{\prime}$ is a switch operator.

$$
\begin{aligned}
\Psi\left(\sigma^{\prime}\right) & =\left(\text { id } \wedge \nu \sigma^{\prime}\right) \vee \sigma^{\prime} \nu \\
& =(\text { id } \wedge \nu(\sigma \eta \wedge \text { id })) \vee(\sigma \eta \wedge \text { id }) \nu \\
& =(\text { id } \wedge(\nu \sigma \eta \vee \nu)) \vee(\sigma \eta \nu \wedge \nu) \\
& =(\text { id } \wedge \nu \sigma \eta) \vee(\sigma \eta \nu \wedge \nu) .
\end{aligned}
$$

Now we substitute that $\sigma=\Sigma(\psi)=\mathrm{id} \wedge \psi \nu$, and use that $\nu \eta \leq \nu \leq \eta \nu$ :

$$
\begin{aligned}
\Psi\left(\sigma^{\prime}\right) & =(\text { id } \wedge \nu(\text { id } \wedge \psi \nu) \eta) \vee((\mathrm{id} \wedge \psi \nu) \eta \nu \wedge \nu) \\
& =(\text { id } \wedge(\nu \vee \psi) \eta) \vee\left(\left(\eta \nu \wedge \psi \eta^{*}\right) \wedge \nu\right) \\
& =((\mathrm{id} \wedge \nu \eta) \vee(\mathrm{id} \wedge \psi \eta)) \vee\left(\psi \eta^{*} \wedge \nu\right) \\
& =(\text { id } \wedge \psi \eta) \vee\left(\nu \wedge \psi \eta^{*}\right)
\end{aligned}
$$

Since $\eta^{*} \leq \eta$ we have id $\wedge \psi \eta^{*} \leq$ id $\wedge \psi \eta$; thus

$$
\begin{aligned}
\Psi\left(\sigma^{\prime}\right) & =(\mathrm{id} \wedge \psi \eta) \vee\left(\eta \wedge \psi \eta^{*}\right) \vee\left(\mathrm{id} \wedge \psi \eta^{*}\right) \\
& =(\mathrm{id} \wedge \psi \eta) \vee \psi \eta^{*}
\end{aligned}
$$

This concludes the first part of the assertion.

Using (S1) and the extensivity of $\eta$ we get that $X \cap$ $\sigma(\eta(X)) \subseteq \sigma(X)$ for every $X$, hence $\sigma^{\prime} \leq \sigma$. Now Proposition 3.3 gives that $\psi^{\prime} \preccurlyeq \psi$. This concludes the proof.

From the following section onwards we will restrict ourselves to operators which are translation invariant. 
Before we do so we will present a class of self-dual operators (and their corresponding switch operators) based on the notion of adjacency.

Let $E$ be an arbitrary non-empty set and let $\sim$ be a symmetric relation on $E \times E$. Thus $x \sim y$ iff $y \sim x$, for $x, y \in E$. In this case we say that $x$ and $y$ are adjacent. The set of points adjacent to $x$ is denoted by $\operatorname{adj}(x)$

$$
\operatorname{adj}(x)=\{y \in E \mid x \sim y\} .
$$

N.B. It is not assumed that $\sim$ is reflexive; hence $x \sim x$ may not be satisfied.

When the set $E$ is finite we can supply it with a graph structure by considering the unordered pairs $\{x, y\}$ with $x \sim y$ as edges. We define the operator

$$
\sigma(X)=\{h \in X \mid \operatorname{adj}(h) \neq \emptyset \text { and } \operatorname{adj}(h) \cap X=\emptyset\} .
$$

\section{Proposition 4.4. $\sigma$ is a switch operator.}

Proof: First we prove (S1). Let $X \subseteq Y$; we show that $X \cap \sigma(Y) \subseteq \sigma(X)$. If $x \in X \cap \sigma(Y)$, then $x \in Y$ and $\operatorname{adj}(x) \cap Y=\emptyset$. This implies that $\operatorname{adj}(x) \cap X=\emptyset$, too, hence $x \in \sigma(X)$.

To prove (S2), assume that $h \in \sigma(X \cup\{h\}) \cap \sigma\left(X^{c} \cup\right.$ $\{h\})$. Then $\operatorname{adj}(h) \neq \varnothing$ and $\operatorname{adj}(h) \cap(X \cup\{h\})=\emptyset$ and $\operatorname{adj}(h) \cap\left(X^{c} \cup\{h\}\right)=\emptyset$. But this yields that $\operatorname{adj}(h)=\emptyset$, a contradiction.

This concludes the proof.

From this point onwards we assume that

$$
\operatorname{adj}(h) \neq \emptyset, \quad \text { for every } h \in E \text {. }
$$

Let $\psi$ be the increasing, self-dual operator on $\mathcal{P}(E)$ associated with $\sigma$. The action of $\psi$ can be described as follows: $\psi$ switches the state of a point if and only

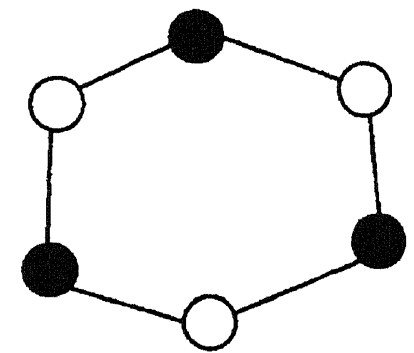

Figure 5. A period-2 orbit. if all its adjacent points have a different state. Or, to put it differently, $\psi$ switches the state of the isolated points. Therefore, if $x, y$ are adjacent and have the same state, then both points are left unaltered by $\psi$ (and its subsequent iterates).

Define the dilation $\delta$ by

$$
\delta(X)=\{h \in E \mid \operatorname{adj}(h) \cap X \neq \varnothing\} .
$$

The negative erosion $\varepsilon=\delta^{*}$ is then given by

$$
\varepsilon(X)=\{h \in E \mid \operatorname{adj}(h) \subseteq X\} .
$$

Note that these two operators are known from the context of graph morphology [6].

Evidently, the pair $(\varepsilon, \delta)$ constitutes an adjunction. It is easy to check that $\nu \sigma=\nu \vee \delta$ and that $\sigma \nu=\nu \wedge \varepsilon$. Substitution in $\psi=($ id $\wedge \nu \sigma) \vee \sigma \nu$ gives us

$$
\psi=(\mathrm{id} \wedge \delta) \vee(\nu \wedge \varepsilon) .
$$

Using that $\varepsilon \leq \delta$ we get id $\wedge \varepsilon \leq \mathrm{id} \wedge \delta$, and therefore

$$
\psi=(\mathrm{id} \wedge \delta) \vee(\nu \wedge \varepsilon) \vee(\mathrm{id} \wedge \varepsilon),
$$

that is

$$
\psi=(\mathrm{id} \wedge \delta) \vee \varepsilon=(\mathrm{id} \vee \varepsilon) \wedge \delta .
$$

We present a condition on the adjacency relation which guarantees that the operator $\psi$ is idempotent. In general, however, $\psi$ will not be idempotent. In Fig. 5 for example, we depict a binary image on a graph whose edges represent the adjacency relation, which shows period 2 oscillations under iteration of $\psi$.

Definition 4.5. Let $x, y, z \in E$; we call $\{x, y, z\}$ an adjacency triple if $x \sim y \sim z \sim x$.

The points $x, y, z$ need not be distinct. In fact, if $x \sim x$, then $\{x, x, x\}$ is an adjacency triple.

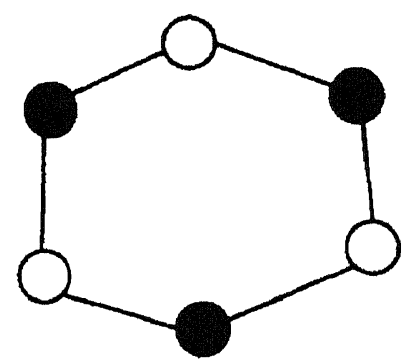


The following intuitive argument shows that $\psi$ is idempotent if every point in $E$ is a member of at least one adjacency triple. Suppose namely that point $x \in X$ has no adjacent points inside $X$. Since it is a member of an adjacency triple $\{x, y, z\}$ with $y, z \in X^{c}$, the point $x$ will be mapped outside $X$ by $\psi$. However, the points $y, z$ stay inside $X^{c}$ because they constitute an adjacent pair. In subsequent iterations the points $x, y, z$ will stay in $X^{c}$.

Proposition 4.6. Assume that every point in $E$ is a member of at least one adjacency triple, then $\psi$ is idempotent.

Proof: We show that $\psi \leq \psi^{2}$. Then, taking the negative at both sides and using that $\psi^{*}=\psi$ we get $\psi \geq \psi^{2}$ as well.

Let $h \in \psi(X)$. There are two alternatives: $h \in X$ and $h \notin X$. Assume first that $h \in X$; then $h \notin \sigma(X)$, which means that $\operatorname{adj}(h) \cap X \neq \emptyset$. Let $x \in \operatorname{adj}(h) \cap X$, then $h \in \operatorname{adj}(x) \cap X$, and therefore $x \notin \sigma(X)$. We conclude that $x \in \psi(X)$. But then $h \notin \sigma(\psi(X))$, meaning that $h \in \psi^{2}(X)$.

Assume next that $h \notin X$. Then $h \in \sigma\left(X^{c}\right)$ and thus $\operatorname{adj}(h) \cap X^{c}=\emptyset$. By assumption, $h$ is a member of some adjacency triple: $h \sim x \sim y \sim h$. Necessarily, $x, y \in X$. But then $h, x, y \in \psi(X)$ and it follows that $h \in \psi^{2}(X)$ (as well as $x, y \in \psi^{2}(X)$ ).

This concludes the proof.

It is obvious that the adjacency triple condition is not satisfied in the example of Fig. 5.

Let us apply this result to the case that $E=\mathbb{Z}^{2}$ (or $\mathbb{Z}^{d}$ ), where $\sim$ is translation invariant, i.e., $x \sim y$ implies that $x+h \sim y+h$ for all $x, y, h \in \mathbb{Z}^{2}$. Putting $A=\operatorname{adj}(0)$, we have $\operatorname{adj}(h)=A_{h}$. The fact that the adjacency relation is symmetric implies that $A$ is symmetric; i.e., $A=\check{A}$, where $\breve{A}=\{-a \mid a \in A\}$. The adjacency triple condition can be formulated as follows:

$$
0 \in A \oplus A \oplus A
$$

Furthermore

$$
\delta(X)=X \oplus A, \varepsilon(X)=X \ominus A .
$$

We arrive at the following result.

Theorem 4.7. Let $A \subseteq \mathbb{Z}^{2}$ be a symmetric structuring element $(A=\check{A})$ which satisfies

$$
0 \in A \oplus A \oplus A \text {. }
$$

Then the increasing, translation invariant operator $\psi$ given by

$$
\psi(X)=(X \cap(X \oplus A)) \cup(X \ominus A)
$$

is self-dual and idempotent.

Note that the conditions on $A$ are satisfied by

$$
A=\left[\begin{array}{lll}
\bullet & \bullet & \bullet \\
\bullet & \vdots & \bullet \\
\bullet & \bullet & \bullet
\end{array}\right.
$$

The operator $\psi$ in Theorem 4.7 switches all pixels whose state is different from that of all their 8-neighbours (i.e., the isolated pixels); see also Example 10 in Section 7.

\section{Switch Operators Which are Translation Invariant}

Arriving at this point, the most obvious question is: how to design switch operators? Below we shall give a complete characterization of translation invariant switch operators. But first we give a characterization of the kernel of a translation invariant self-dual operator. Recall that the kernel of a translation invariant operator $\psi$ on $\mathcal{P}\left(\mathbb{Z}^{2}\right)$ is defined as $[13,3]$ :

$$
\mathcal{V}(\psi)=\left\{A \subseteq \mathbb{Z}^{2} \mid 0 \in \psi(A)\right\}
$$

Every increasing, translation invariant operator $\psi$ can be written as a union of erosions:

$$
\psi(X)=\bigcup_{A \in \mathcal{V}(\psi)} X \ominus A .
$$

Recall also that [3]

$$
\mathcal{V}\left(\bigwedge_{i \in I} \psi_{i}\right)=\bigcap_{i \in I} \mathcal{V}\left(\psi_{i}\right)
$$

and

$$
\mathcal{V}\left(\bigvee_{i \in I} \psi_{i}\right)=\bigcup_{i \in I} \mathcal{V}\left(\psi_{i}\right)
$$

Proposition 5.1. Let $\psi$ be a translation invariant operator; then $\psi$ is self-dual if and only if

$$
A \in \mathcal{V}(\psi) \Longleftrightarrow A^{c} \notin \mathcal{V}(\psi)
$$


If, moreover, $\psi$ is increasing, then

$$
A, B \in \mathcal{V}(\psi) \Rightarrow A \cap B \neq \emptyset .
$$

Proof: $A \in \mathcal{V}(\psi)$ iff $0 \in \psi(A)=\psi^{*}(A)=$ $\left(\psi\left(A^{c}\right)\right)^{c}$. This implies the first assertion.

Assume that $\psi$ is also increasing. If $A, B \in \mathcal{V}(\psi)$ and $A \cap B=\emptyset$, then $A \subseteq B^{c}$. This implies that $0 \in \psi(A) \subseteq \psi\left(B^{c}\right)$, a contradiction.

Assume that $\psi$ is an increasing, translation invariant, self-dual operator, and let $\sigma=\Sigma(\psi)$. Using (5.1) we get

$$
\sigma(X)=\bigcup_{A \in \mathcal{V}(\psi)} X \cap\left(X^{c} \ominus A\right)
$$

If $0 \in A$ then $X \cap\left(X^{c} \ominus A\right)=\emptyset$, and thus we find

$$
\sigma(X)=\bigcup_{A \in \mathcal{V}(\psi), 0 \notin A} X \cap\left(X^{c} \ominus A\right) .
$$

Using $\sigma=$ id $\wedge \psi \nu$ and (5.2) we obtain $\mathcal{V}(\sigma)=$ $\mathcal{V}$ (id) $\cap \mathcal{V}(\psi \nu)$. This is equivalent to

$A \in \mathcal{V}(\psi), 0 \notin A \Longleftrightarrow 0 \in \sigma\left(A^{c}\right) \Longleftrightarrow A^{c} \in \mathcal{V}(\sigma)$.

Define

$$
a(\sigma)=\left\{A \subseteq \mathbb{Z}^{2} \mid A^{c} \in \mathcal{V}(\sigma)\right\} .
$$

Proposition 5.1 gives that $A \cap B \neq \emptyset$ for $A, B \in a(\sigma)$. We have thus shown the following result.

Proposition 5.2. Let $\sigma$ be a switch operator and let $a(\sigma)$ be given by (5.3); then

$$
0 \notin A \text { and } A \cap B \neq \emptyset \text {, for } A, B \in a(\sigma) \text {. }
$$

Furthermore, $\sigma$ is given by

$$
\sigma(X)=X \cap \bigcup_{A \in a(\sigma)} X^{c} \ominus A .
$$

The following question arises. If $\psi$ is an increasing, translation invariant, self-dual operator, then $\psi$ can be represented as a supremum of erosions, $\psi=$ $\bigvee_{A \in \mathcal{V}(\psi)} \varepsilon_{A}$, where $\varepsilon_{A}$ is the erosion $\varepsilon_{A}(X)=X \ominus A$. The corresponding switch operator $\sigma=\Sigma(\psi)$ is characterized completely by a subset of $\mathcal{V}(\psi)$, namely those structuring elements which do not contain the origin. It is easy to see that $\psi \geq \bigvee_{A \in a(\sigma)} \varepsilon_{A}$; in general, equality does not hold. Yet the collection $a(\sigma)$ is sufficiently large to recover $\psi$. How can we express $\psi$ in terms of this collection?

More generally, let $\mathcal{A}$ be a collection of structuring elements which obey

$$
0 \notin A \text { and } A \cap B \neq \emptyset, \text { for } A, B \in \mathcal{A} \text {. }
$$

Let

$$
\sigma(X)=\sigma_{\mathcal{A}}(X)=X \cap \bigcup_{A \in \mathcal{A}} X^{c} \ominus A,
$$

that is,

$$
\sigma_{\mathcal{A}}=\mathrm{id} \wedge \bigvee_{A \in \mathcal{A}} \varepsilon_{A} \nu
$$

To show that $\sigma$ is a switch operator, we compute $\psi_{\mathcal{A}}=\Psi\left(\sigma_{\mathcal{A}}\right)$ and show that it is an increasing, selfdual operator.

$$
\begin{aligned}
\psi_{\mathcal{A}} & =\left(\mathrm{id} \wedge \nu \sigma_{\mathcal{A}}\right) \vee \sigma_{\mathcal{A}} v \\
& =\left(\mathrm{id} \wedge \bigwedge_{A \in \mathcal{A}} \delta_{\check{A}}\right) \vee\left(\nu \wedge \bigvee_{A \in \mathcal{A}} \varepsilon_{A}\right) .
\end{aligned}
$$

Since $A \cap B \neq \emptyset$ for $A, B \in \mathcal{A}$ we get that $\delta_{\check{A}} \geq \varepsilon_{B}$ for $A, B \in \mathcal{A}$. This implies that id $\wedge \wedge_{A \in \mathcal{A}} \delta_{\check{A}} \geq$ id $\wedge \bigvee_{A \in \mathcal{A}} \varepsilon_{A}$, which leads us to

$$
\begin{aligned}
\psi_{\mathcal{A}} & =\left(\mathrm{id} \wedge \bigwedge_{A \in \mathcal{A}} \delta_{\check{A}}\right) \vee\left(\nu \wedge \bigvee_{A \in \mathcal{A}} \varepsilon_{A}\right) \vee\left(\mathrm{id} \wedge \bigvee_{A \in \mathcal{A}} \varepsilon_{A}\right) \\
& =\left(\mathrm{id} \wedge \bigwedge_{A \in \mathcal{A}} \delta_{\check{A}}\right) \vee \bigvee_{A \in \mathcal{A}} \varepsilon_{\dot{A}} .
\end{aligned}
$$

In other words, $\psi_{\mathcal{A}}$ is the centre of the increasing operator $\bigvee_{A \in \mathcal{A}} \varepsilon_{A}$ and its negation $\bigwedge_{A \in \mathcal{A}} \delta_{\check{A}}$.

Theorem 5.3. Every translation invariant switch operator $\sigma$ is of the form $\sigma=\sigma_{\mathcal{A}}$, where $\mathcal{A}$ is a collection of structuring elements satisfying (5.6). The corresponding self-dual operator $\psi_{\mathcal{A}}=\Psi\left(\sigma_{\mathcal{A}}\right)$ is given by

$$
\psi_{\mathcal{A}}(X)=\left(X \cap \bigcap_{A \in \mathcal{A}} X \oplus \check{A}\right) \cup \bigcup_{A \in \mathcal{A}} X \ominus A .
$$


If $\sigma$ is a switch operator and $\mathcal{A}$ a collection of structuring elements satisfying (5.6) such that $\sigma=\sigma_{\mathcal{A}}$, then we say that $\sigma$ is generated by $\mathcal{A}$. Note that, in general, $\mathcal{A}$ is a subcollection of $a(\sigma)$. To compute the set $a\left(\sigma_{\mathcal{A}}\right)$ observe that

or

$$
B \in \mathcal{V}\left(\psi_{\mathcal{A}}\right) \Longleftrightarrow B \in \mathcal{V}\left(\mathrm{id} \wedge \bigwedge_{A \in \mathcal{A}} \delta_{\check{A}}\right)
$$

$$
B \in \mathcal{V}\left(\bigvee_{A \in \mathcal{A}} \varepsilon_{A}\right)
$$

Elements $B \in \mathcal{V}\left(\right.$ id $\left.\wedge \bigwedge_{A \in \mathcal{A}} \delta_{\breve{A}}\right)$ contain the origin, and as such they are not contained in $a\left(\sigma_{\mathcal{A}}\right)$. Furthermore, $B \in \mathcal{V}\left(\bigvee_{A \in \mathcal{A}^{A}} \varepsilon_{A}\right)$ yields that $A \subseteq B$ for some $A \in \mathcal{A}$. Thus we conclude that

$$
a\left(\sigma_{\mathcal{A}}\right)=\{B \mid 0 \notin B \text { and } A \subseteq B \text { for some } A \in \mathcal{A}\}
$$

A structuring element $B \in \mathcal{A}$ is redundant if there exists another structuring element $A \in \mathcal{A}$ which is a strict subset of $B$.

Note that $\sigma_{\mathcal{A}}$ can also be written as a union of hit-ormiss operators, namely

$$
\sigma_{\mathcal{A}}(X)=\bigcup_{A \in \mathcal{A}} X \circledast(\{0\}, A) .
$$

We conclude this section with the following result.

\section{Proposition 5.4.}

(a) Let $\sigma, \sigma^{\prime}$ be translation invariant switch operators; then

$$
\sigma \leq \sigma^{\prime} \Rightarrow a(\sigma) \subseteq a\left(\sigma^{\prime}\right)
$$

(b) Let $\mathcal{A}, \mathcal{B}$ be subsets of $\mathcal{P}\left(\mathbb{Z}^{2}\right)$. If $\mathcal{A} \subseteq \mathcal{B}$ then $\sigma_{\mathcal{A}} \leq \sigma_{\mathcal{B}}$

(c) The following two assertions are equivalent:

(i) $\sigma_{\mathcal{A}} \leq \sigma_{\mathcal{B}}$;

(ii) for every $A \in \mathcal{A}$ there exists a $B \in \mathcal{B}$ such that $B \subseteq A$.

Proof: (a) and (b) are obvious (in fact, (b) is a consequence of (c)). We prove (c). First assume (i) and let $A \in \mathcal{A}$. Then $0 \in A^{c}$ and $0 \in \sigma_{\mathcal{A}}\left(A^{c}\right)$. As $\sigma_{\mathcal{A}} \leq \sigma_{\mathcal{B}}$, we conclude that $0 \in \sigma_{\mathcal{B}}\left(A^{c}\right)$; so there exists a $B \in \mathcal{B}$ such that $B \subseteq A$. This proves (ii)

The proof that (ii) $\Rightarrow$ (i) is straightforward.
Corollary 5.5. If $\mathcal{A}, \mathcal{B}$ are subsets of $\mathcal{P}\left(\mathbb{Z}^{2}\right)$ such that

(i) $\mathcal{B} \subseteq \mathcal{A}$;

(ii) for every $A \in \mathcal{A}$ there exists a $B \in \mathcal{B}$ such that $B \subseteq A$;

then $\sigma_{\mathcal{A}}=\sigma_{\mathcal{B}}$.

Example 5.6. Consider the operators $\theta_{5}, \theta_{6}, \ldots, \theta_{9}$ defined in Example 3.1, and define $\sigma_{k}=\Sigma\left(\theta_{k}\right)$, $k=5, \ldots, 9$. Then $\sigma_{k}$ is generated by the collection $\mathcal{A}_{k}$ of subsets $A$ of the $3 \times 3$ square centred at the origin with $0 \notin A$ and $\operatorname{card}(A)=k$. This collection $\mathcal{A}_{k}$ contains $\left(\begin{array}{l}8 \\ k\end{array}\right)$ subsets (which equals 0 if $k=9$ ). For example, $\mathcal{A}_{6}$ consists of 28 structuring elements:

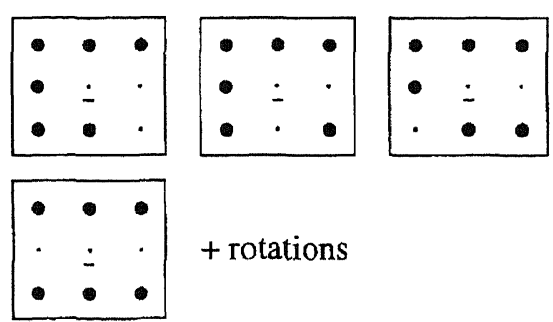

Refer to Section 6, Examples 7 and 8, for some additional results concerning this example.

\section{Construction of Self-Dual Filters}

In $[12,5,3]$ it has been shown how to construct morphological filters by iteration. We recall the following result.

Proposition 6.1. Let $\psi$ be an increasing, finitewindow operator, let $\psi^{\infty}$ be an increasing operator, and assume that $\psi^{n} \rightarrow \psi^{\infty}$ (pointwise) as $n \rightarrow \infty$. Then $\psi^{\infty}$ is a morphological filter.

It is obvious that the sequence $\psi^{n}$ converges to a limit if $\psi$ is extensive or anti-extensive. In Section 2 we have seen that an activity-extensive operator $\psi$ has the property that every sequence $\psi^{n}(X)$ is pointwise monotone, and hence convergent. Therefore, Proposition 6.1 applies to operators which are activity-extensive.

Suppose that $\psi$ is an increasing, translation invariant, self-dual operator. Is there a simple way to verify whether or not $\psi$ is activity-extensive? A simple, but very useful, criterion is given by the following result.

Proposition 6.2. Let $\psi$ be an arbitrary operator, and assume that there exists a set $X$ such that $\psi(X) \neq X$ 
and $\psi^{p}(X)=X$ for some integer $p>1$. Then $\psi$ is not activity-extensive.

In this case we call $X$ periodic with respect to $\psi$. If $p>1$ is the smallest integer for which $\psi^{p}(X)=$ $X$ then we say that $X$ is $p$-periodic, or alternatively, that $X$ has period $p$. For example, the chess board is 2 -periodic with respect to the median operator which uses the rhombus (origin and its four 4-neighbours) as structuring element.

Let $\psi$ be an increasing, translation invariant, selfdual operator with $\Sigma(\psi)=\sigma_{\mathcal{A}}$. If $A \in \mathcal{A}$, then $0 \notin$ $A$ and $0 \in \psi(A)$. If there exists an integer $p>1$ such that $0 \notin \psi^{p}(A)$, then the sequence $\psi^{n}(A)$ is not pointwise monotone, and therefore $\psi$ is not activityextensive. On the other hand, suppose that $0 \in \psi^{n}(A)$ for every $n \geq 1$ and $A \in \mathcal{A}$. We show that $\psi$ is activityextensive. Suppose, namely, that $\psi$ is not activityextensive. Then there exists a set $X$ such that $\psi^{n}(X)$ is not pointwise monotone. Without loss of generality we can assume that $0 \notin X, 0 \in \psi(X)$, and $0 \notin \psi^{p}(X)$ for some integer $p>1$. Thus $0 \in \sigma\left(X^{c}\right)$, which means that $A \subseteq X$ for some $A \in \mathcal{A}$. But then, by assumption, $0 \in \psi^{n}(A) \subseteq \psi^{n}(X)$ for every $n \geq 1$, a contradiction. We have proved the following result.

Proposition 6.3. Let $\psi$ be an increasing, translation invariant, self-dual operator with switch operator $\sigma_{\mathcal{A}}$. Then $\psi$ is activity-extensive if and only if $0 \in \psi^{n}(A), n \geq 1$, for every $A \in \mathcal{A}$.

In practice, the structuring elements in $\mathcal{A}$ are finite and the sequence $\psi^{n}(A)$ becomes stationary (i.e., constant) after finitely many iterations. In such cases, verification of the condition in Proposition 6.3 requires only finitely many computations.

Example 6.4. (a) The operator $\theta_{6}$ defined in Example 3.1 is not activity-extensive since

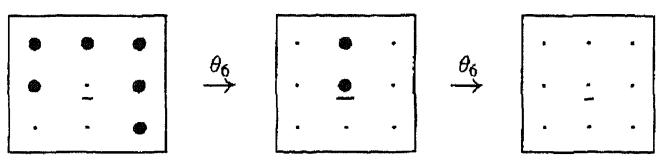

(b) The operator $\theta_{7}$ is activity-extensive, since

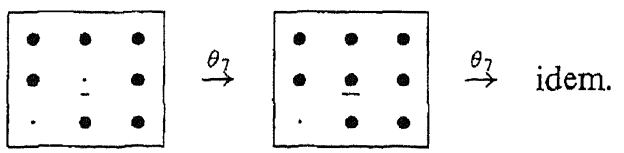

and

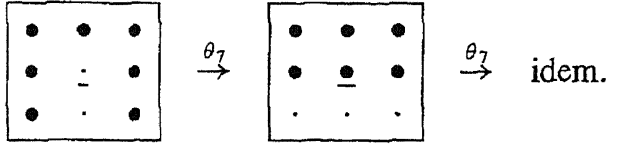

The other structuring elements in $\mathcal{A}_{7}$ are $90^{\circ}$-rotations of these two elements.

In [4] and [3] we have explained how one can modify an increasing, self-dual operator which is not activityextensive in such a way that it becomes activityextensive. In fact this approach is based on the following two results.

Proposition 6.5 (See [14] and [3, Chapter 13]). Assume that $\phi, \psi$ are finite-window operators, $\phi \leq \psi$, and that $\phi$ is an inf-overfilter and $\psi$ a sup-underfilter. Then the centre $\gamma=(\mathrm{id} \wedge \psi) \vee \phi$ is activity-extensive and the sequence $\gamma^{n}$ converges to the strong middle filter of $\phi$ and $\psi$.

Proposition 6.6 (See [3, Proposition 6.28]). Let $\psi$ be an increasing operator.

(a) If $\alpha$ is an opening with $\alpha \leq \psi$ then $\psi \alpha$ is an infoverfilter

(b) If $\beta$ is a closing with $\beta \geq \psi$ then $\psi \beta$ is a supunderfilter.

If we combine the previous two results with Proposition 4.3 we arrive at the following construction method for self-dual morphological filters.

Proposition 6.7 (Construction of Self-Dual Filters). Let $\psi$ be an increasing, self-dual operator with switch operator $\sigma=\Sigma(\psi)$. Let $\alpha$ be an opening $\leq \psi$, and let $\beta=\alpha^{*}$ be the negative closing. Then

$$
\pi=(\operatorname{id} \wedge \psi \beta) \vee \psi \alpha
$$

is an increasing self-dual operator with $\Sigma(\pi)=\mathrm{id} \wedge$ $\sigma \beta$. The operator $\pi$ is activity-extensive and satisfies $\pi \preccurlyeq \psi$.

If the operators $\psi, \alpha, \beta$ are finite-window operators, then $\pi$ is a finite-window operator too, and $\pi^{n}$ converges towards a strong self-dual morphological filter $\pi^{\infty}$. (In fact, $\pi^{\infty}$ is the middle filter of $\psi \alpha$ and $\psi \beta$.)

This result makes clear that we must look for openings $\alpha$ which are $\leq \psi$. Recall that the structural opening 
$\alpha_{B}$ is given by $\alpha_{B}(X)=X \circ B$. It is a well-known fact [3] that

$$
\alpha_{B} \leq \psi \quad \text { iff } \quad \psi(B) \geq B .
$$

The following result characterizes all structuring elements $B$ with this property.

Proposition 6.8. Assume that $\psi$ is increasing and self-dual, and that the switch operator $\sigma=\Sigma(\psi)$ can be represented as $\sigma=\sigma_{\mathcal{A}}$, for some collection $\mathcal{A} \subseteq$ $\mathcal{P}\left(\mathbb{Z}^{2}\right)$. The following assertions are equivalent:

(i) $\psi(B) \geq B$;

(ii) $\sigma(B)=\emptyset$;

(iii) $\forall b \in B \forall A \in \mathcal{A}: A_{b} \cap B \neq \emptyset$;

(iv) $\forall A \in \mathcal{A}: 0 \in \check{A} \bullet B$.

\section{Proof:}

(i) $\Longrightarrow$ (ii): using (3.1) we get

$$
\begin{aligned}
\sigma(B)=\emptyset & \Longleftrightarrow B \cap \psi\left(B^{c}\right)=\emptyset \\
& \Longleftrightarrow \psi\left(B^{c}\right) \subseteq B^{c} \\
& \Longleftrightarrow B \subseteq\left(\psi\left(B^{c}\right)\right)^{c}=\psi^{*}(B)=\psi(B) .
\end{aligned}
$$

(ii) $\Longrightarrow$ (iii): using the expression for $\sigma_{\mathcal{A}}$ in (5.7) we get

$$
\begin{aligned}
\sigma_{\mathcal{A}}(B)=\emptyset & \Longleftrightarrow B \cap \bigcup_{A \in \mathcal{A}} B^{c} \ominus A=\emptyset \\
& \Longleftrightarrow \forall b \in B: b \notin \bigcup_{A \in \mathcal{A}} B^{c} \ominus A \\
& \Longleftrightarrow \forall b \in B \forall A \in \mathcal{A}: b \notin B^{c} \ominus A \\
& \Longleftrightarrow \forall b \in B \forall A \in \mathcal{A}: A_{b} \nsubseteq B^{c} \\
& \Longleftrightarrow \forall b \in B \forall A \in \mathcal{A}: A_{b} \cap B \neq \emptyset .
\end{aligned}
$$

(iii) $\Longleftrightarrow$ (iv): above we have seen that (iii) is equivalent with

$$
\forall b \in B \forall A \in \mathcal{A}: b \notin B^{c} \ominus A .
$$

This implies

$$
\begin{aligned}
\forall b & \in B \forall A \in \mathcal{A}: b \in\left(B^{c} \ominus A\right)^{c}=B \oplus \check{A} \\
& =\check{A} \oplus B \\
\Longleftrightarrow \forall A & \in \mathcal{A}: B \subseteq \check{A} \oplus B \\
\Longleftrightarrow \forall A & \in \mathcal{A}: 0 \in(\check{A} \oplus B) \ominus B=\check{A} \bullet B .
\end{aligned}
$$

This concludes the proof.
A structuring element $B$ which satisfies the equivalent properties in Proposition 6.8 is called persistent with respect to $\psi$. Note that, unless $\psi=\mathrm{id}$, singletons are not persistent. It is obvious that the collection of persistent sets is closed under unions: if $B_{i}$ is persistent for every $i$ in some index set $I$, then $\bigcup_{i \in I} B_{i}$ is persistent, too.

Assume that $\psi, \psi^{\prime}$ are increasing, self-dual operators, and that $\psi^{\prime} \preccurlyeq \psi$. If $B$ is persistent with respect to $\psi$ then it is also persistent with respect to $\psi^{\prime}$.

\section{Examples}

In this section we present several increasing, translation invariant, self-dual operators $\psi$ on $\mathcal{P}\left(\mathbb{Z}^{2}\right)$. All examples contain the following ingredients:

- characterization of a (minimal) collection of structuring elements $\mathcal{A}$ such that $\sigma_{\mathcal{A}}$ is the switch operator associated with $\psi$;

- an examination of the activity-extensivity of $\psi$;

- (in case that $\psi$ is not activity-extensive) characterization of a collection of structuring elements which are persistent with respect to $\psi$.

In the latter case one can use the opening $\alpha(X)=$ $\bigvee_{i \in I} X \circ B_{i}$, where $B_{i}, i \in I$, are persistent structuring elements, to obtain a self-dual operator

$$
\pi=\left(\mathrm{id} \wedge \psi \alpha^{*}\right) \vee \psi \alpha
$$

which is activity-extensive (see Proposition 6.7). It should be evident that our interest goes to "small" persistent structuring elements, as these cause the smallest "loss of activity" if one replaces $\psi$ with $\pi$. The operators $\psi, \pi$ in Example $i$ are denoted by $\psi_{i}, \pi_{i}$, respectively $(i=1,2, \ldots, 13)$

1. Let $A$ be the rhombus comprising the origin and its four horizontal and vertical neighbours. Let $\rho_{A, s}$ be the rank operators defined in Section 3, and let $\psi_{1}$ be the centre of the operator $\rho_{A .2}$ and its dual $\rho_{A, 4}$, i.e., $\psi_{1}=\left(\right.$ id $\left.\wedge \rho_{A, 2}\right) \vee \rho_{A .4}$ (cf. Example 3.1); then $\psi_{1}$ is self-dual. It is easy to verify that $\psi_{1}=\psi_{\mathcal{A}}$, where $\mathcal{A}$ contains one structuring element:

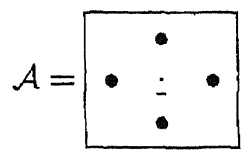


The operator $\psi_{1}$ is not activity-extensive for the chess board is a periodic structure. The elements

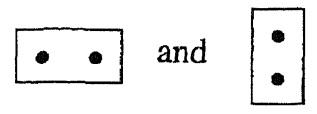

are persistent. The operator $\psi_{1}$ switches the state of all pixels which are isolated in the 4-connected sense. Let $A$ be the structuring element depicted above, then $\psi_{1}$ is given by (4.6). However, the adjacency triple condition governed by (4.5) is not satisfied.

2. Again, let $A$ be the rhombus, and let $\psi_{2}$ be the corresponding median operator. Then $\psi_{2}=\psi_{\mathcal{A}}$ with

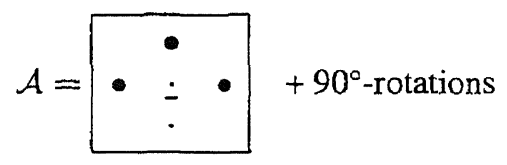

Like in the previous example, $\psi_{2}$ is not activityextensive since the chess board is 2-periodic with respect to $\psi_{2}$. The operator $\psi_{2}$ has an infinite number of persistent elements which cannot be decomposed into smaller parts which are persistent, for example:
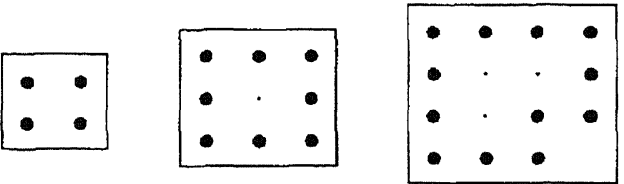

In fact, every structuring element in which every pixel has at least two 4-neighbours is persistent.

3. Consider the median operator $\psi_{3}$ associated with the $3 \times 3$ square. In Section 3 we have depicted the structuring elements in $\mathcal{A}$ such that $\psi_{3}=\psi_{\mathcal{A}}$; we recall them here for the sake of convenience.

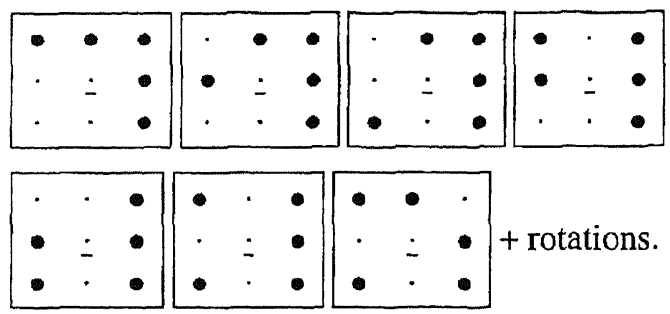

$\psi_{3}$ is not activity-extensive, because the following structure is 2-periodic:

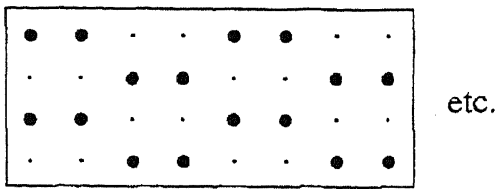

The set

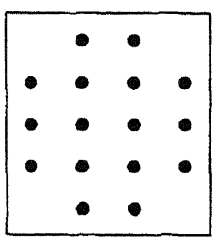

is a persistent structuring element. Let $\alpha$ be the corresponding structural opening, and let $\pi_{3}$ be the modification of $\psi_{3}$ resulting from (7.1). The median operator $\psi_{3}$, its modification $\pi_{3}$, and the filter $\pi_{3}^{\infty}$ obtained by infinite iteration are illustrated in the second row of Fig. 6 .

4. Consider the subcollection $\mathcal{B}$ of $\mathcal{A}$ in Example 3 which lacks the first structuring element along with its three $90^{\circ}$-rotations. The corresponding selfdual operator $\psi_{4}$ is less active than $\psi_{3}$. However, it is not activity-extensive for the structure depicted in 3 is 2-periodic. Of course, the persistent element in 3 is also persistent with respect to $\psi_{4}$, but there also exists a smaller element which does the job, namely

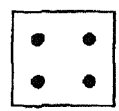

Denote by $\pi_{4}$ the modification of $\psi_{4}$ resulting from (7.1), where $\alpha$ is the opening by this structuring element. Refer to the third row in Fig. 6 for an illustration.

5. Consider the subcollection $\mathcal{C}$ of $\mathcal{A}$ in 3 which lacks the $45^{\circ}, 135^{\circ}, 225^{\circ}$ and $315^{\circ}$-rotations of the first structuring element, i.e., the elements
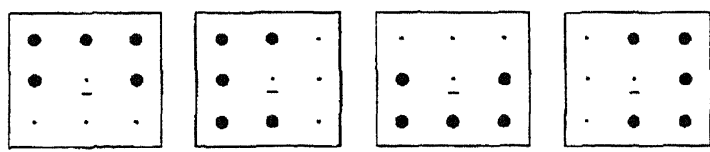

Then $\psi_{5}:=\psi_{c}$ is less active than $\psi_{3}$ but it is not activity-extensive for, again, the structure depicted in 3 is 2-periodic with respect to $\psi_{5}$. In this case 


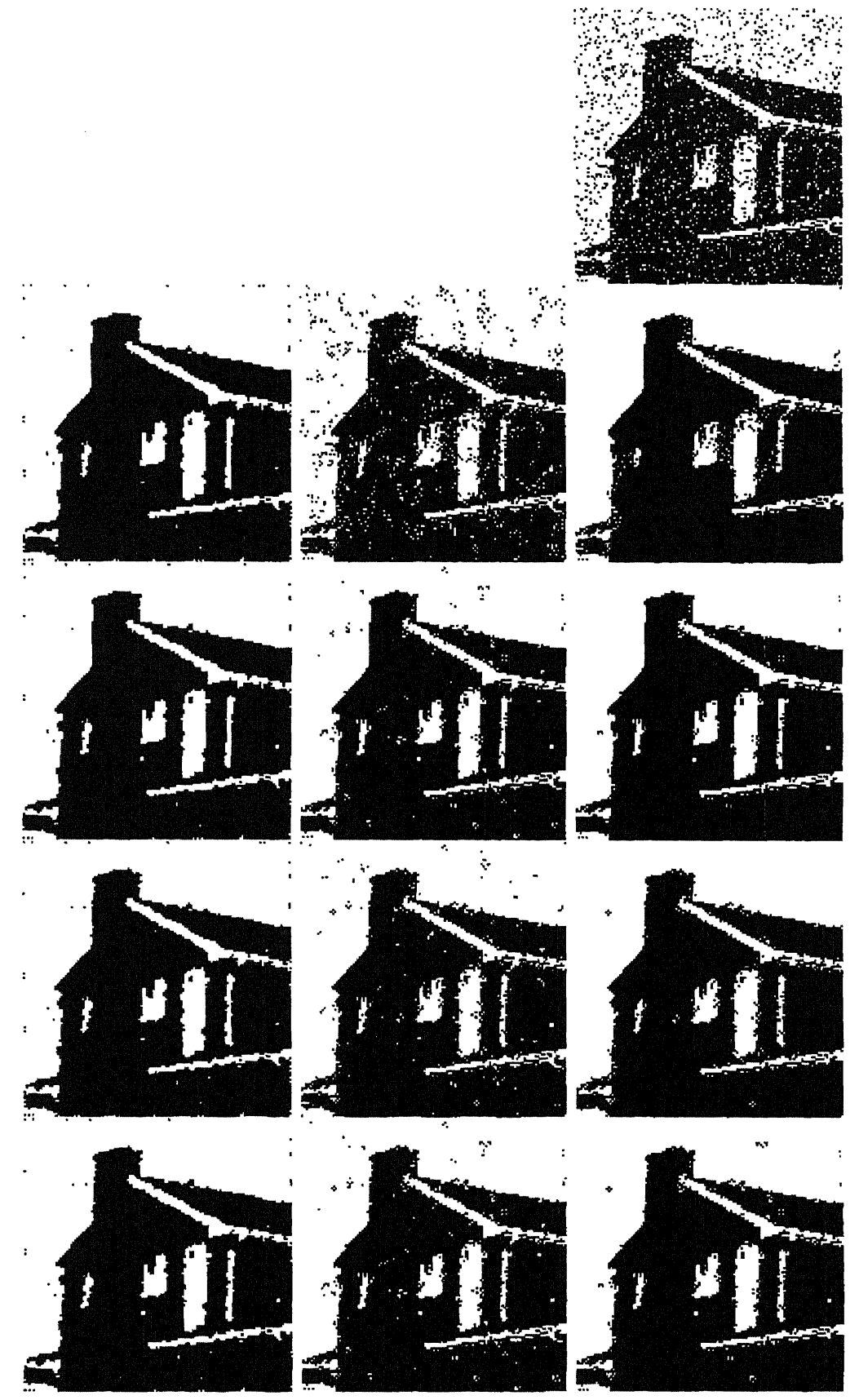

Figure 6. The top-right image is the original image $X$. The four subsequent rows show (from left to right) the images $\psi_{i}(X), \pi_{i}(X), \pi_{i}^{\infty}(X)$ defined in examples $i=3,4,5,6$, respectively. For a better understanding of the usefulness of the modifications $\pi_{i}$ and their iterates one should compare these images with the ones in Fig. 1. 
the rhombus

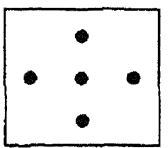

is persistent with respect to $\psi_{5}$.

Denote by $\pi_{5}$ the modification of $\psi_{5}$ resulting from (7.1), where $\alpha$ is the opening by this structuring element. The operators in this example are illustrated in the fourth row of Fig. 6 .

6. We can combine 4 and 5 and consider the subcollection $\mathcal{D}$ of $\mathcal{A}$ which lacks the eight rotations of the first structuring element in $\mathcal{A}$, i.e., $\mathcal{D}=\mathcal{B} \cap \mathcal{C}$. It is relatively easy to show that $\sigma_{\mathcal{D}}=\sigma_{\mathcal{B}} \wedge \sigma_{\mathcal{C}}$ (here one must use that $B \in \mathcal{B}$ and $C \in \mathcal{C}$ implies that $D \subseteq B \cup C$ for some $D \in \mathcal{D}$ ). Now we conclude from (3.11) that $\psi_{6}:=\psi_{\mathcal{D}}$ is the centre of $\psi_{4}=\psi_{\mathcal{B}}$ and $\psi_{5}=\psi_{\mathcal{C}}$ :

$$
\psi_{6}=\psi_{4} \curlywedge \psi_{5}
$$

In this case

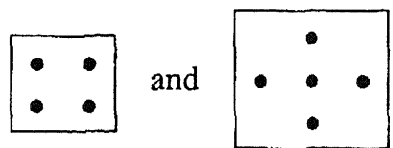

are persistent with respect to $\psi_{6}$. Let $\pi_{6}$ be the modification of $\psi_{6}$ resulting from (7.1) if we take for $\alpha$ the union of the structural openings by these two elements. The action of the operators in this example are depicted in the bottom row of Fig. 6 .

7. Consider again the operator $\theta_{6}$ introduced in Example 3.1; see also Example 5.6. Here we shall denote this operator by $\psi_{7}$. The collection of structuring elements associated with $\Sigma\left(\psi_{7}\right)$ is given by

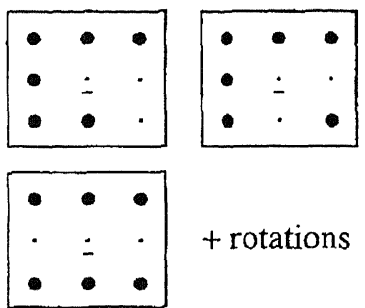

The operator $\psi_{7}$ is not activity-extensive since the pattern

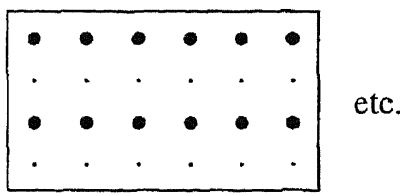

is 2-periodic. The elements

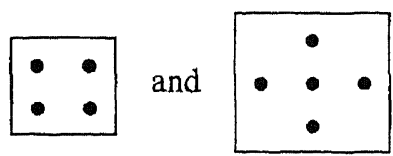

are persistent. Let $\pi_{7}$ be the modification of $\psi_{7}$ obtained from (7.1) if one takes for $\alpha$ the opening by these two structuring elements. The second row in Fig. 7 depicts the action of $\psi_{7}, \pi_{7}$, and $\pi_{7}^{\infty}$.

8. Consider the subcollection $\mathcal{B}$ of $\mathcal{A}_{6}$ which is obtained by deleting the elements
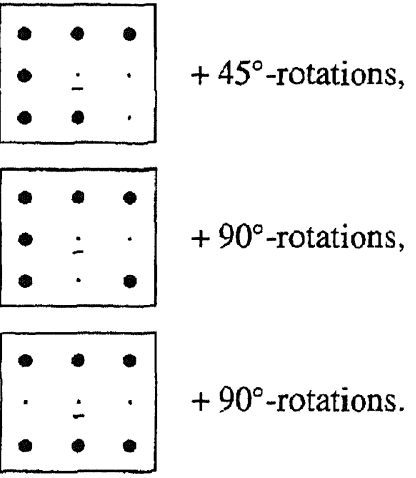

We use Proposition 6.3 to show that $\psi_{8}=\psi_{\mathcal{B}}$ is activity-extensive. Thereto we must consider the sequences $\psi_{8}^{n}(B)$ for $B \in \mathcal{B}$. First we note that the elements

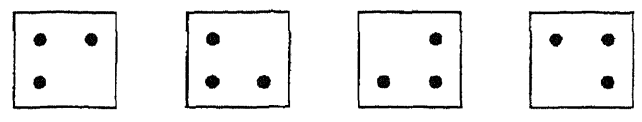

are invariant under $\psi_{8}$. Now

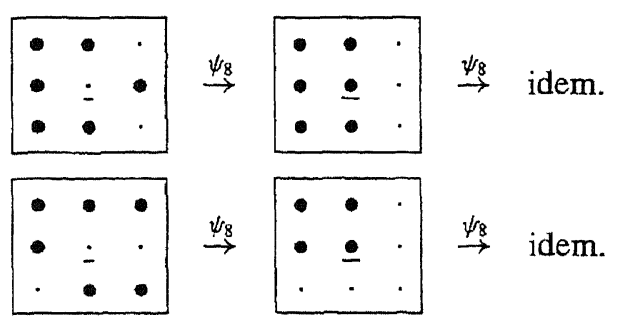




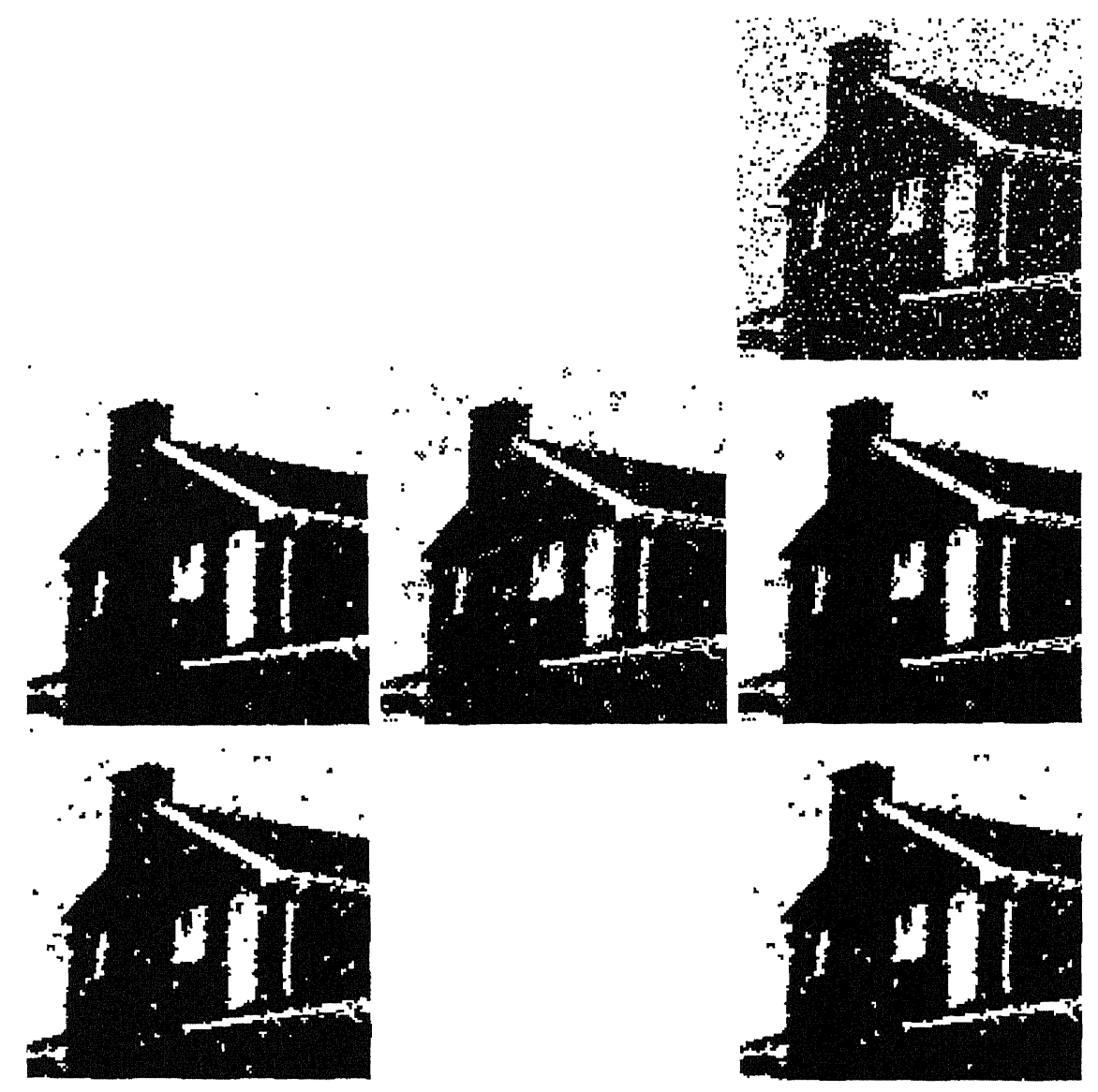

Figure 7. The top-right image is the original image $X$. The second row illustrates the operators $\psi_{7}, \pi_{7}$, and $\pi_{7}^{\infty}$ discussed in Example 7. The bottom row depicts $\psi_{8}(X)$ and $\psi_{8}^{\infty}(X)$ examined in Example 8.

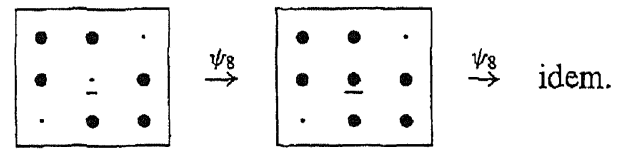

This implies that $\psi_{8}$ is activity-extensive. The bottom row in Fig. 7 depicts the transformed sets $\psi_{8}(X)$ (left) and $\psi_{8}^{\infty}(X)$ (right). These figures show clearly the invariance of the triangles depicted above.

9. Consider the operator $\theta_{7}$ introduced in Example 3.1. We define $\psi_{9}:=\theta_{7}$. The associated collection $\mathcal{A}_{7}$ consists of eight structuring elements:

$$
\mathcal{A}_{7}=\left[\begin{array}{lll}
\bullet & \bullet & \bullet \\
\hdashline & \vdots & \bullet \\
\bullet & \bullet & \cdot
\end{array}\right. \text { + rotations }
$$

Using Proposition 6.3 it is easy to show that $\psi_{9}$ is activity-extensive. Use namely that

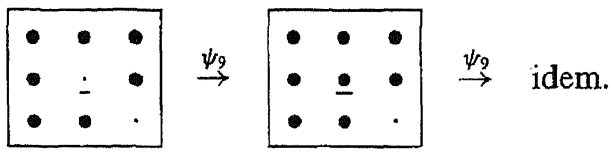

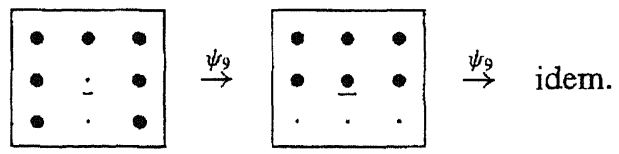

In Fig. 8 we depict $X, \psi_{9}(X)$ and $\psi_{9}^{\infty}(X)$.

10. Consider the operator $\psi_{10}:=\theta_{8}$; here $\mathcal{A}_{8}$ contains one structuring element, namely

$$
\mathcal{A}_{8}=\begin{array}{lll}
\bullet & \bullet & \bullet \\
\bullet & \vdots & \bullet \\
\bullet & \bullet & \bullet \\
\hline
\end{array}
$$




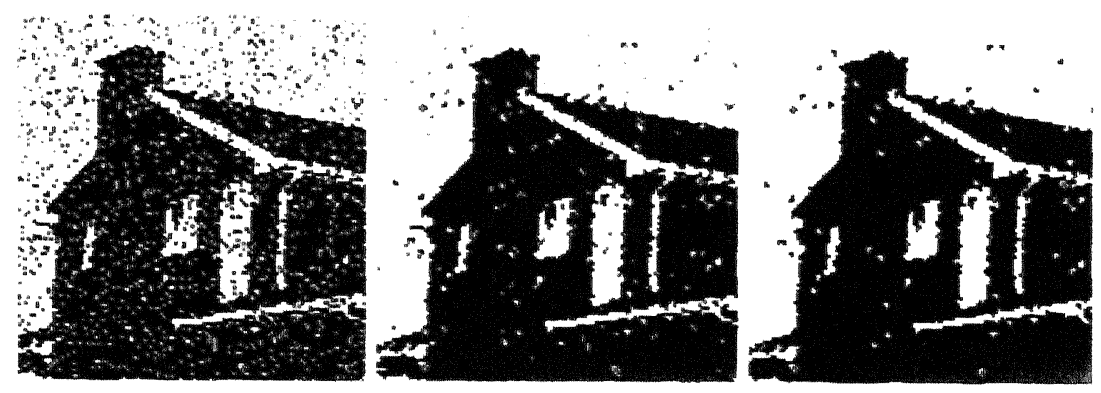

Figure 8. From left to right: $X, \psi_{9}(X)$ and $\psi_{9}^{\infty}(X)$; see Example 9

The structuring element in $\mathcal{A}_{8}$ satisties condition (4.5) in Theorem 4.7, meaning that the resulting operator $\psi_{10}$ is idempotent. Its action consists hereof that it removes isolated foreground and background pixels (isolated in the sense of 8 neighbours). An illustration can be found in Fig. 2.

11. Consider the weighted median operator $\psi_{11}$ with matrix

$$
\left(\begin{array}{lll}
1 & 3 & 1 \\
3 & 5 & 3 \\
1 & 3 & 1
\end{array}\right)
$$

and threshold 11. The collection of structuring elements $\mathcal{A}$ yielding the corresponding switch operator is
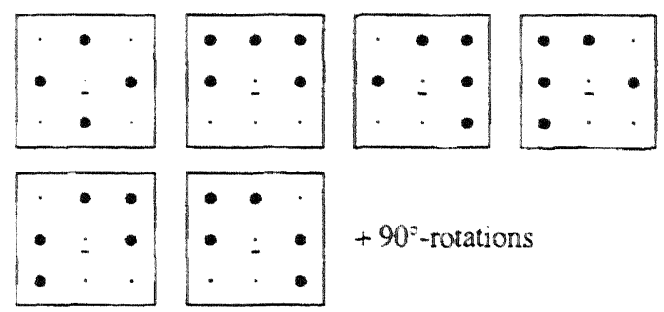

$+90^{\circ}$-rotations

If $\mathcal{B}$ is the collection of structuring elements associated with the rhombus median operator (see Example 2) then condition (ii) in Proposition 5.4(c) is satisfied, hence the operator $\psi_{11}$ is less active than the rhombus median operator. The chess board is periodic with respect to $\psi_{11}$ and therefore $\psi_{11}$ is not activity-extensive. The pattern

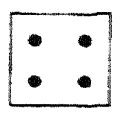

is persistent. Let $\pi_{11}$ be the resulting activityextensive modification of $\psi_{11}$. In Fig. 9, second row, we depict $\psi_{11}(X), \pi_{11}(X)$, and $\pi_{11}^{\infty}(X)$.
12. Let $\rho$ be the weighted rank operator obtained by changing the threshold in the previous example to 9. Let $\psi_{12}$ be the centre of $\rho$ and its negative $\rho^{*}$. It is evident that this operator is less active than the one in 11 . The switch operator $\Sigma\left(\psi_{12}\right)$ is generated by the structuring elements:

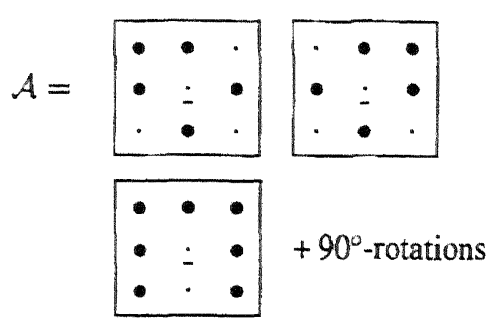

We use Proposition 6.3 to show that $\psi_{12}$ is activityextensive:

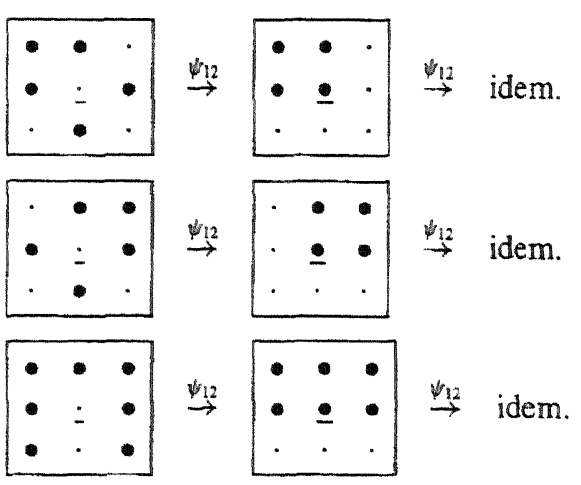

This implies that $\psi_{12}$ is activity-extensive. Observe that the elementary triangles

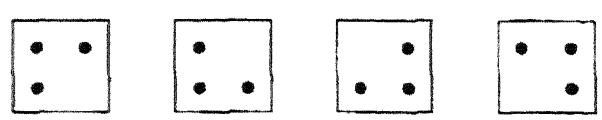

are persistent. In the third row in Fig. 9 we depict $\psi_{12}(X)$ (left) and $\psi_{12}^{\infty}(X)$ (right). These images 


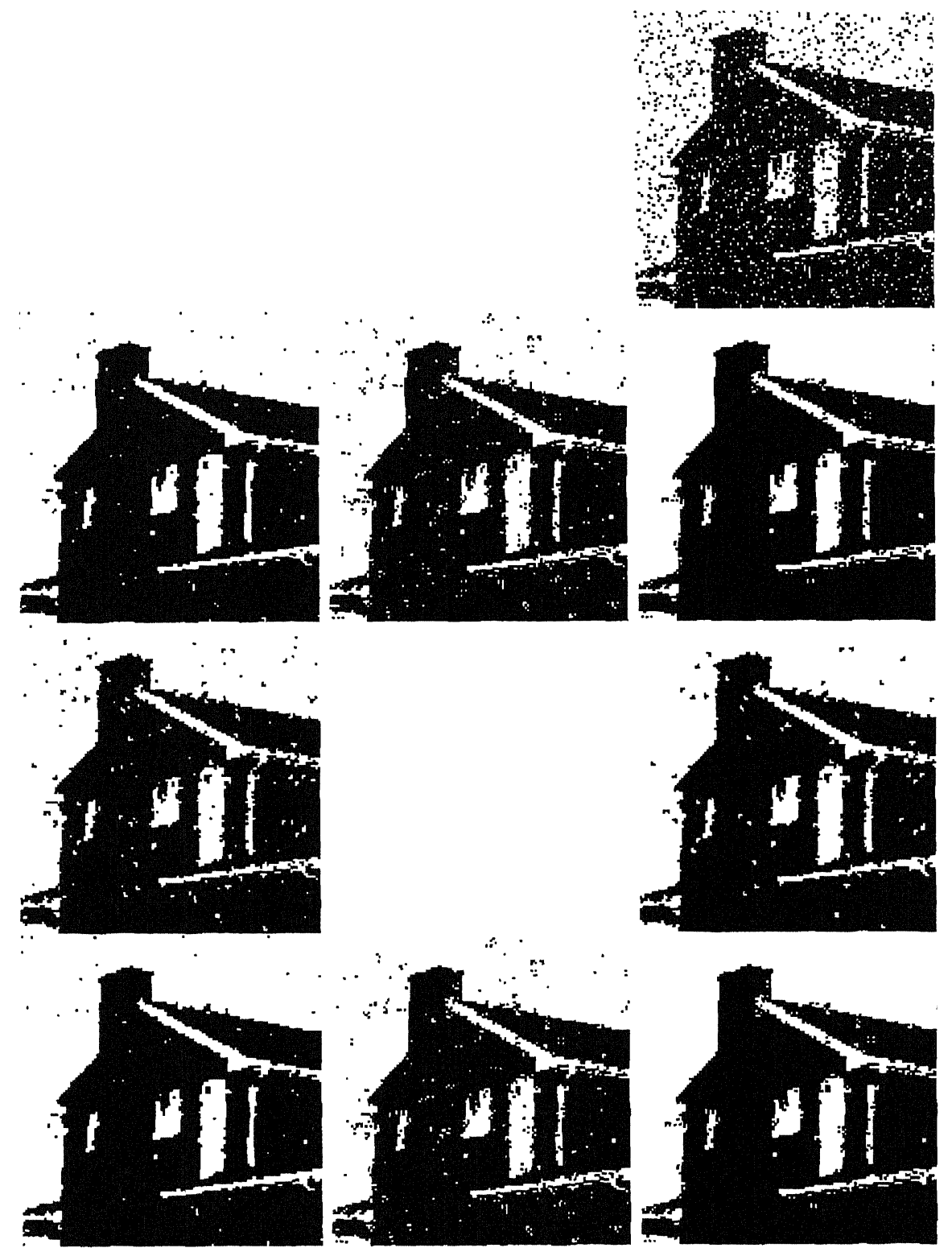

Figure 9. The top-right image is the original image $X$. The second row illustrates the operators $\psi_{11}, \pi_{11}$, and $\pi_{11}^{\infty}$. The third row illustrates $\psi_{12}$ and $\psi_{12}^{\infty}$, and the bottom row depicts the operators $\psi_{13}, \pi_{13}$, and $\pi_{13}^{\infty}$.

show clearly the invariance of the elementary triangles. In this respect this example resembles the one in Example 8.

13. If we add

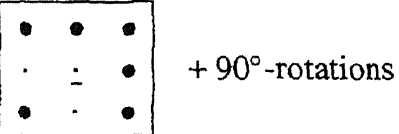

to the collection $\mathcal{A}$ in 11 we obtain an operator $\psi_{13}$ which is more active than the one in 11 . Note that this operator corresponds with the weighted median with matrix

$$
\left(\begin{array}{lll}
1 & 2 & 1 \\
2 & 3 & 2 \\
1 & 2 & 1
\end{array}\right)
$$


and threshold 8. The chess board is periodic and therefore $\psi_{13}$ is not activity-extensive. Again

$$
\bullet \bullet
$$

is persistent. Let $\pi_{13}$ be the corresponding activityextensive modification of $\psi_{13}$. In the bottom row of Fig. 9 we depict $\psi_{13}(X), \pi_{13}(X)$, and $\pi_{13}^{\infty}(X)$.

\section{Conclusions and Open Problems}

The basic morphological operators like dilations, erosions, openings, closings, and alternating sequential filters are not self-dual. The best known morphological operator which is self-dual is the median operator $\mu$. Unfortunately, this operator has a property which is undesirable from the view point of noise filtering: the sequence of iterates $\mu^{n}(X)$ of an input image $X$ needs not be convergent. In fact, it is easy to find images $X$ such that $\{X, \mu(X)\}$ constitutes a period-2 orbit.

In this paper two goals have been accomplished. First, we have presented a general method, based on the concept of a switch operator, for the construction of increasing operators which are self-dual. Under the extra assumption of translation invariance we have derived a general formula for self-dual operators:

$$
\psi_{\mathcal{A}}(X)=\left(X \cap \bigcap_{A \in \mathcal{A}} X \oplus \check{A}\right) \cup \bigcup_{A \in \mathcal{A}} X \ominus A,
$$

where $\mathcal{A}$ is an arbitrary collection of structuring elements satisfying

$$
0 \notin A \quad \text { and } \quad A \cap B \neq \varnothing, \text { for } A, B \in \mathcal{A} \text {. }
$$

In this paper we have restricted ourselves to binary images for simplicity. Yet, it is obvious that formula (8.1) carries over immediately to grey-scale images, and in fact, this remark applies to most of the results established in this paper.

A second accomplishment is that we have derived a general method for the modification of an arbitrary increasing, self-dual operator $\psi$ yielding another increasing, self-dual operator $\pi$ which is less active than $\psi$, but which has the desirable property that it is activityextensive. This means in particular that $\pi^{n}$ converges to a self-dual morphological filter $\pi^{\infty}$ (presumed that it is a finite-window operator).
We have illustrated our theoretical results with a large number of examples. We have limited ourselves to the square grid, and we have only considered structuring elements which fit inside a $3 \times 3$ window. Furthermore, the structuring elements have been chosen in such a way that the resulting operator, besides being translation invariant, is invariant under rotations about 90 degrees. From a theoretical point of view there is no need to make such restrictions. In fact, in an earlier paper [2] we have investigated modifications of the median operator on the hexagonal grid, such as the Maisonneuve filter [9]; see also [3, Example 13.59].

We have resisted the temptation to make quantitative statements about the noise cleaning capacities of the operators and filters discussed in our examples, others than those in terms of the activity ordering. For example the illustration of Example 8 in Fig. 7 and Example 12 in Fig. 9 show that $\psi_{8}^{\infty}(X)$ is very similar to $\psi_{12}^{\infty}(X)$, though both images differ at a few points. The only theoretical support for this resemblance is that both operators keep elementary triangles unchanged.

The research described here also raises a number of intriguing theoretical questions which fall outside the scope of this paper, but which, nevertheless deserve to be mentioned in this final section.

Question 1. Do there exist operators which have orbits with period greater than 2 ?

Period-3 orbits may be of special interest because of the possible analogy with the famous result obtained by $\mathrm{Li}$ and Yorke [8] in the context of dynamical systems theory which says that "period three implies chaos".

The following question is related to the previous one.

Question 2. Given an increasing, translation invariant (self-dual) operator $\psi$ (e.g. the median operator). What kind of behaviour can the orbits $\left\{X, \psi(X), \psi^{2}(X), \ldots\right\}$ exhibit? Do they necessarily "converge" to a periodic orbit? Can such morphological dynamical systems exhibit "chaotic behaviour"?

These appear to be difficult questions which require a lot of fundamental research. Maybe one can find (partial) answers from the literature on cellular automata [15]. To our knowledge however, the overlap between these two fields, that is, mathematical morphology on the one hand and cellular automata on the other, is almost completely unexplored up till today; refer to [11] for some related discussions. Hopefully, the theory developed in this paper will serve as a stimulus to fill this gap. 


\section{References}

1. G.J.F. Banon and J. Barrera, "Minimal representation for translation-invariant set mappings by mathematical morphology," SIAM J. Appl. Math., Vol. 51, pp. 1782-1798, 1991.

2. H.J.A.M. Heijmans, "Morphological filtering and iteration," in Visual Communications and Image Processing'90, Vol. 1360 of SPIE Proceedings, Lausanne, 1990, pp. 166-175.

3. H.J.A.M. Heijmans, Morphological Image Operators, Academic Press: Boston, 1994.

4. H.J.A.M. Heijmans, "On the construction of morphological operators which are selfdual and activity-extensive," Signal Processing, Vol. 38, pp. 13-19, 1994.

5. H.J.A.M. Heijmans and J. Serra, "Convergence, continuity and iteration in mathematical morphology." Journal of Visual Communications and Image Representation, Vol. 3, pp. 84-102, 1992.

6. H.J.A.M. Heijmans and $\mathbf{L}$. Vincent, "Graph morphology in image analysis," in Mathematical Morphology in Image Processing, E.R. Dougherty (Ed.), Marcel Dekker: New York, 1993, Ch. 6, pp. 171-203.

7. B.I. Justusson, "Median filtering: statistical properties," in TwoDimensional Digital Signal Processing II: Transforms and Median Filters, T.S. Huang (Ed.), Springer-Verlag: Berlin, 1981, pp. 161-196.

8. T.Y. Li and J.A. Yorke, "Period three implies chaos," Amer. Math Monthly, Vol. 82, pp. 985-992, 1975.

9. F. Maisonneuve, "Ordinaux transfinis et sur- (ou sous-) potentes," Technical Report N780, Ecole Nationale Supérieure des Mines de Paris, 1982.

10. G. Matheron, Random Sets and Integral Geometry, John Wiley and Sons: New York, 1975

11. K. Preston, Jr., M.J.B. Duff, S. Levialdi, P.E. Norgren, and J.I. Toriwaki, "Basics of cellular logic with some applications in medical image processing," Proc. IEEE, Vol. 67, pp. 826-856, 1979.
12. C. Ronse and H.J.A.M. Heijmans, "The algebraic basis of mathematical morphology-Part II: Openings and closings," Computer Vision, Graphics and Image Processing: Image Understanding, Vol. 54, pp. 74-97, 1991.

13. J. Serra, Image Analysis and Mathematical Morphology, Academic Press: London, 1982.

14. J. Serra (Ed.), Image Analysis and Mathematical Morphology, II: Theoretical Advances, Academic Press: London, 1988.

15. S. Wolfram (Ed.), Theory and Applications of Cellular Automata: Including Selected Papers 1983-1986, World Scientific: Singapore, 1986.

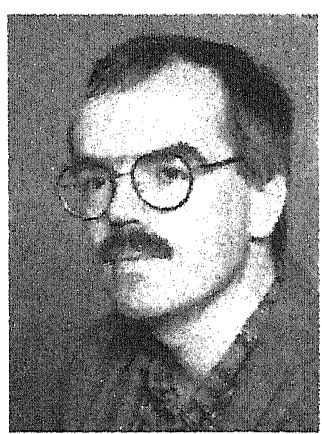

Henk Heijmans received his M.Sc. degree in mathematics from the Technical University of Eindhoven, The Netherlands in 1981. He then joined the Centrefor Mathematics and Computer Science (CWI) in Amsterdam where he worked on mathematical biology, dynamical systems theory and functional analysis. In 1985 he received his $\mathrm{PhD}$ degree from the University of Amsterdam. A year later he became involved in a project on image analysis and mathematical morphology. His primary research interests concern mathematical morphology discrete topology and geometry, and stochastic geometry. 